\title{
Enzyme analysis
}

\section{Murray Logan}

September 26, 2015

\section{Table of contents}

1 Preparations $\mathbf{1}$

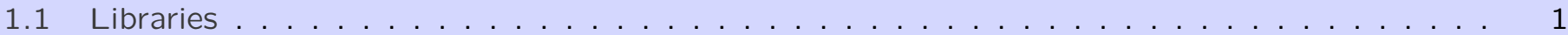

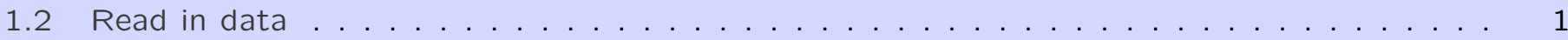

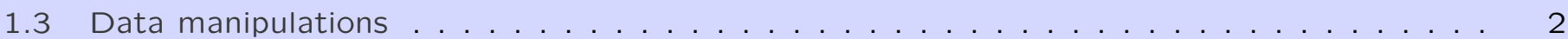

2 Exploratory data analysis 3

3 Analyses 18

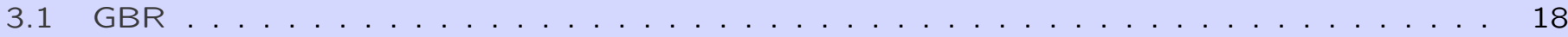

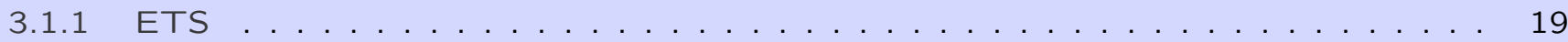

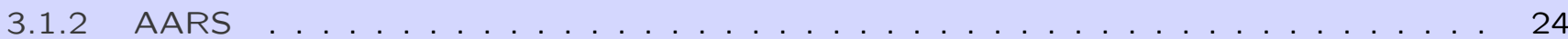

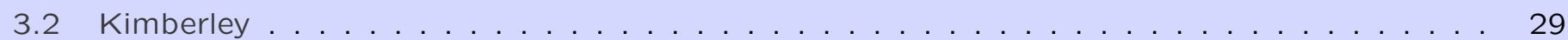

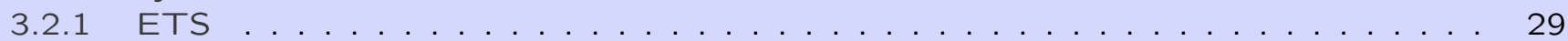

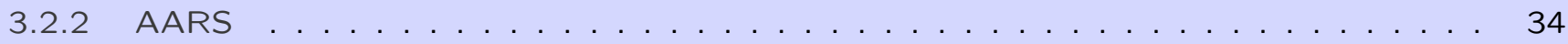

4 References 39

\section{Preparations}

\subsection{Libraries}

$>$ library (ggplot2)

$>$ library (gridExtra)

$>$ library (R2jags)

$>$ library (plyr)

library (xtable)

\subsection{Read in data}

csc <- read.csv('data/CSCzymes.csv', strip.white=TRUE)

$\operatorname{str}(\csc )$

'data.frame': 105 obs. of 8 variables:

\$ Station: Factor w/ 33 levels "CSC042", "CSC043", . : 122345678910 ..

\$ Cruise : int $\begin{array}{lllllllllllllll}1 & 1 & 1 & 1 & 1 & 1 & 1 & 1 & 2 & 2 & \ldots\end{array}$

$\$$ Mesh : int $73737373737373737373 \ldots$

\$ Temp : num $22.92121 .42220 .2 \ldots$

$\begin{array}{lllllllllllll}\$ \mathrm{Chl} & \text { : num } & 0.238 & 0.215 & 0.271 & 0.228 & 0.154 & \ldots\end{array}$

\$ Protein: num $0.1747 \quad 0.2828 \quad 0.0822 \quad 0.2548 \quad 0.0553 \ldots$

$\begin{array}{lllllllllll}\$ \text { ETS }: \text { num } & 63.8 & 157 & 183.7 & 232.6 & 217.7 & \ldots\end{array}$

\$ AARS : num $50.5 \quad 41.4 \quad 27.5 \quad 33.6 \quad 61.1 \ldots$

$\operatorname{summary}(\mathrm{csc})$

Station CSC075 : 9 CSC042: 3 $\mathrm{CSC} 043: 3$ $\mathrm{CSC} 044: 3$

\section{Cruise}

Min. $\quad: 1.000$

1st Qu.:2.000

Median : 3.000

Mean $: 2.571$
Mesh

Min. : 73

1st Qu.: 73

Median : 150

Mean : :191

Temp
Min. $\quad: 16.57$
1st Qu. $: 21.40$
Median $: 22.25$
Mean $\quad: 23.03$

Min

$\mathrm{Ch} 1$

Median :0.2094

Mean $\quad: 0.2262$

\section{Protein}

Min. $\quad: 0.007117$

1st Qu.:0.177098

Median :0.394378

Mean : : 0.648345 


\begin{tabular}{|c|c|c|c|c|c|}
\hline $\mathrm{CSC} 045: 3$ & 3rd Qu.:4.000 & 3rd Qu.: 350 & 3rd Qu.:24.62 & 3rd Qu.:0.2652 & 3rd Qu.:0.810647 \\
\hline $\begin{array}{l}\text { CSC046 : } 3 \\
\text { (Other) : } 81\end{array}$ & $: 4.000$ & $: 350$ & $: 28.19$ & Max. $\quad: 0.5765$ & $\begin{array}{ll}\text { Max. } & : 5.696086 \\
\text { NA's } & : 2\end{array}$ \\
\hline
\end{tabular}
ETS AARS

$\begin{array}{lrlr}\text { Min. } \quad: 63.8 & \text { Min. } & : 19.71 \\ \text { 1st Qu. :260.9 } & \text { 1st Qu. : } 55.09 \\ \text { Median }: 349.1 & \text { Median }: 77.58 \\ \text { Mean }: 345.6 & \text { Mean }: 87.98 \\ \text { 3rd Qu. }: 440.7 & \text { 3rd Qu. }: 106.83 \\ \text { Max. }: 729.4 & \text { Max. }: 273.69 \\ \text { NA's }: 1 & & \end{array}$

$>$ kim <- read.csv('data/KIMzymes.csv', strip.white=TRUE)

$>\operatorname{str}(\mathrm{kim})$

'data.frame': 267 obs. of 8 variables:

\$ Station: Factor w/ 89 levels "KIM002", "KIM003", . : 11111222223334 ..

\$ Cruise : int $\begin{array}{lllllllllllllllllll}1 & 1 & 1 & 1 & 1 & 1 & 1 & 1 & 1 & 1 & \ldots\end{array}$

$\$$ Mesh : int $73 \quad 15035073 \quad 15035073 \quad 150 \quad 35073 \ldots$

\$ Temp : num $30303030.430 .4 \ldots$

$\$$ Chl : num $3.373 .37 \quad 3.373 .093 .09 \ldots$

\$ Protein: num 1.9986 .097 NA $0.7243 .772 \ldots$

\$ETS : num $428504 \quad 174 \quad 486450 \ldots$

\$ AARS : num $47.9 \quad 38.959 .3 \quad 48.7 \quad 57.7 \quad \ldots$

$\operatorname{summary}(\mathrm{kim})$

\section{Station Cruise $\quad$ Mesh $\quad$ Temp $\quad$ Chl}

KIM002 : 3 Min. : 1.000 Min. : 73 Min. :26.10 Min. :0.3914 Min. : 0.02508

KIM003: 3 1st Qu.:2.000 1st Qu.: 73 1st Qu.:28.52 1st Qu.:0.5476 1st Qu.: 0.48979

KIM004: 3 Median :3.000 Median :150 Median :29.96 Median :0.8060 Median : 1.00035

KIM005: 3 Mean :2.876 Mean :191 Mean :29.37 Mean :1.1126 Mean : 2.57076

KIM007 : 3 3rd Qu.:4.000 3rd Qu.:350 3rd Qu.:30.35 3rd Qu.:1.5562 3rd Qu.: 1.99886

KIM008: 3 Max. :5.000 Max. :350 Max. :31.71 Max. :3.3707 Max. :161.92120

(Other) : 249

ETS AARS

Min. : 86.2 Min. : 12.61

1st Qu.: 397.2 1st Qu.: 83.72

Median : 533.3 Median :126.69

Mean : 574.0 Mean : 136.98

3rd Qu.: 707.8 3rd Qu.:182.47

Max. :2521.5 Max. $: 394.57$

NA's :5 NA's :4

\subsection{Data manipulations}

csc\$Mesh <- factor (csc\$Mesh)

$>\csc \$$ Cruise <- factor (csc\$Cruise)

$>$ kim\$Mesh <- factor(kim\$Mesh)

kim\$Cruise <- factor(kim\$Cruise) 


\section{Exploratory data analysis}

g1<-ggplot (csc, aes $(y=E T S, x=r e p(1, \operatorname{nrow}(\csc )), \operatorname{color}=$ Mesh $))+$ geom_jitter ()$+$

geom_boxplot (aes $(x=2)$, alpha $=0.2)+$

facet_wrap ( 'Cruise, scales='fixed', nrow $=1)+$ theme_classic ()$+$

scale_y_continuous('Activity of enzyme ETS')+

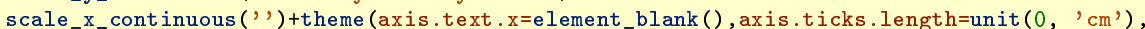

legend . position $=c(0,1)$, legend. justification $=c(0,1)$,

axis.title . $y=e l$ lement_text (vjust=2), plot .margin=unit $(c(0,0,2,2)$, 'lines'))

$\mathrm{g} 2<-\operatorname{ggplot}(\csc , \operatorname{aes}(\mathrm{y}=\mathrm{AARS}, \mathrm{x}=\mathrm{rep}(1, \operatorname{nrow}(\csc )), \operatorname{color}=\operatorname{Mesh}))+$

geom_jitter ()$+$

geom_boxplot $($ aes $(x=2)$, alpha $=0.2)+$

facet_wrap ( Cruise, scales='fixed', nrow $=1)+$ theme_classic ()$+$

scale_y_continuous ('Activity of enzyme AARS') +

scale_x_continuous (' ') +theme (axis.text. $x=e l$ ement_blank (), axis.ticks.length=unit (0, 'cm'),

legend.position $=c(0,1)$, legend.justification $=c(0,1)$,

axis.title . $y=e l$ ement_text (vjust $=2)$, plot .margin=unit $(c(0,0,2,2)$, 'lines'))

grid.arrange $(\mathrm{g} 1, \mathrm{~g} 2, \mathrm{nrow}=2)$

g1<-ggplot (kim, aes $(y=E T S, x=r e p(1$, nrow $($ kim $))$, color=Mesh $))+$ geom_jitter ()$+$

geom_boxplot (aes $(\mathrm{x}=2)$, alpha $=0.2)+$

facet_wrap ( Cruise, scales=' fixed', nrow $=1)+$ theme_classic ()$+$

scale_y_continuous('Activity of enzyme ETS') +

scale_x_continuous("') +theme (axis.text. $x=$ element_blank(), axis.ticks.length=unit (0, 'cm'),

legend.position $=c(0,1)$, legend.justification $=c(0,1)$,

axis.title. $y=$ element_text (vjust=2), plot.margin=unit $(c(0,0,2,2)$, 'lines'))

g2<-ggplot (kim, aes $(y=A A R S, x=r e p(1, \operatorname{nrow}(k i m)), \operatorname{color}=$ Mesh $))+$

geom_jitter ()$+$

geom_boxplot $($ aes $(\mathrm{x}=2)$, alpha $=0.2)+$

facet_wrap ( 'Cruise, scales='fixed',nrow $=1)$ +theme_classic ()$+$

scale_y_continuous('Activity of enzyme AARS') +

scale_x_continuous (', ) +theme (axis.text. $x=$ element_blank (), axis.ticks.length=unit ( 0 , 'cm'),

legend. position $=c(0,1)$, legend.justification $=c(0,1)$,

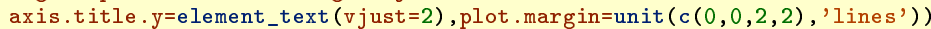

grid. arrange ( $\mathrm{g} 1, \mathrm{~g} 2, \mathrm{nrow}=2)$

g1 <- ggplot(csc, aes (y=ETS, x=Temp, color=Mesh)) + geom_point()+facet_wrap( ${ }^{2}$ Cruise, scales='fixed',nrow=1)+geom_smooth(method='lm')+theme_classic()

g2 - ggplot(csc, aes (y=AARS, $x=$ Temp, color=Mesh)) + geom_point ()+facet_wrap ( ${ }^{2}$ Cruise, scales='fixed', nrow=1)+geom_smooth(method='lm')+theme_classic

grid. arrange ( $\mathrm{g} 1, \mathrm{~g} 2, \mathrm{nrow}=2)$

g1 <- ggplot(kim, aes (y=ETS, $x=$ Temp, color=Mesh)) + geom_point()+facet_wrap( ${ }^{2}$ Cruise, scales='fixed',nrow=1)+geom_smooth(method='lm')+theme_classic()

g2 <- ggplot(kim, aes (y=AARS, $x=$ Temp, color=Mesh)) + geom_point()+facet_wrap( ${ }^{2}$ Cruise, scales='fixed', nrow=1)+geom_smooth(method='lm')+theme_classic

grid . arrange $(\mathrm{g} 1, \mathrm{~g} 2, \mathrm{nrow}=2)$

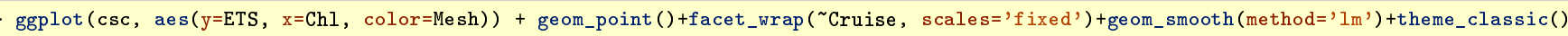

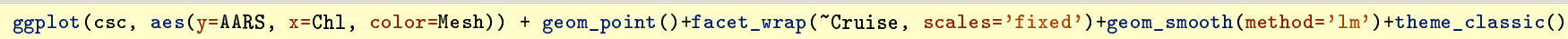

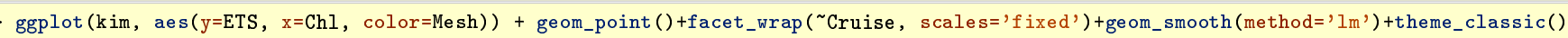

ggplot (kim, aes (y=AARS, $x=C h l$, color=Mesh)) + geom_point()+facet_wrap( (Cruise, scales='fixed')+geom_smooth(method='lm')+theme_classic()

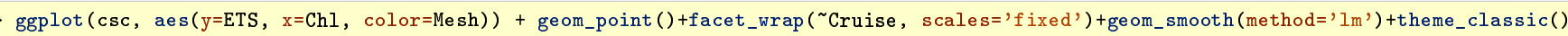

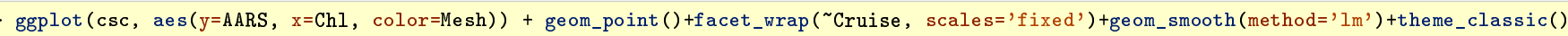

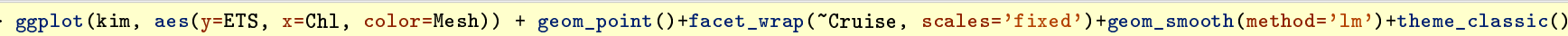

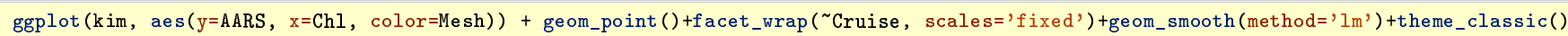



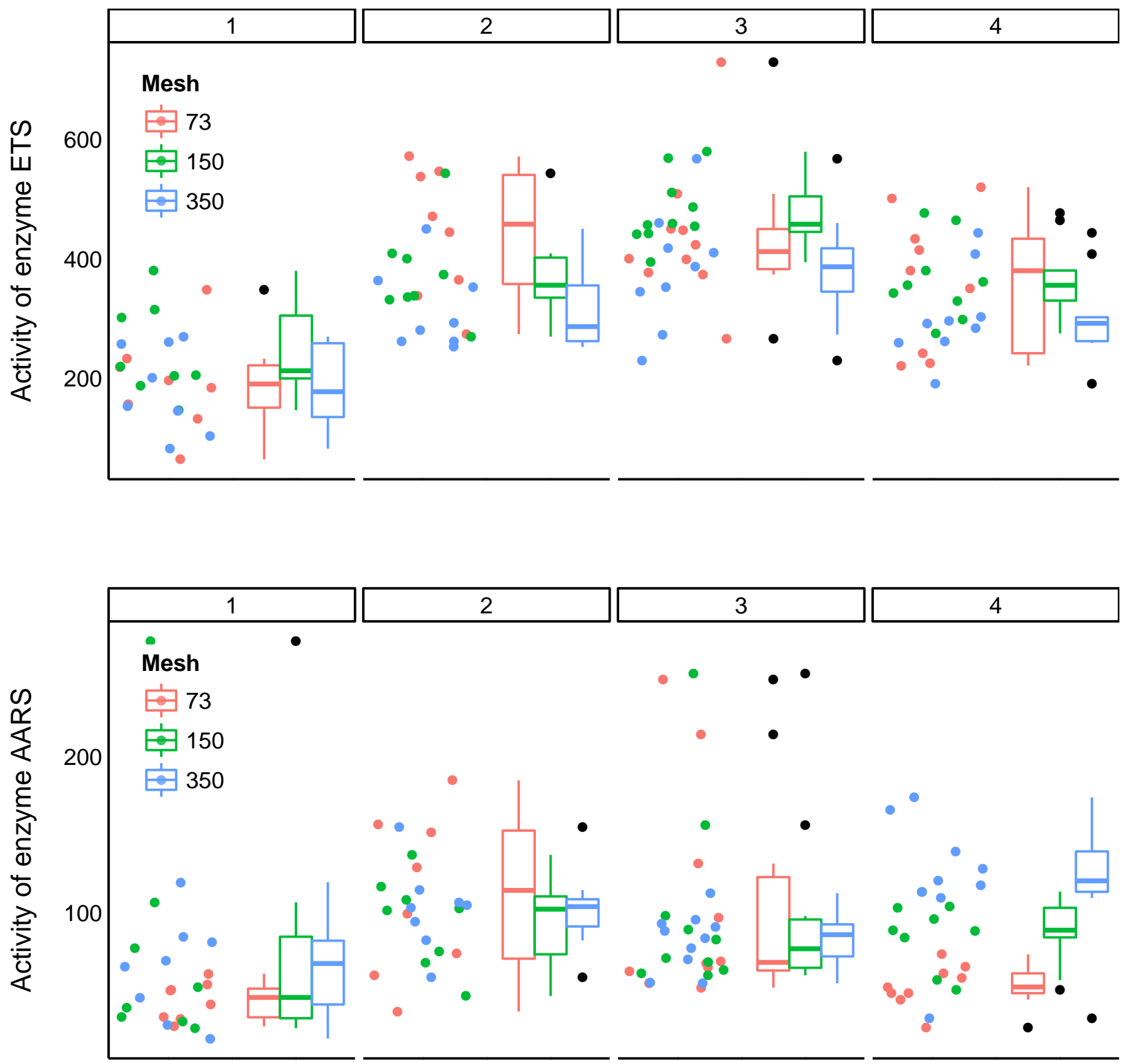

Figure A. plot of chunk eda 

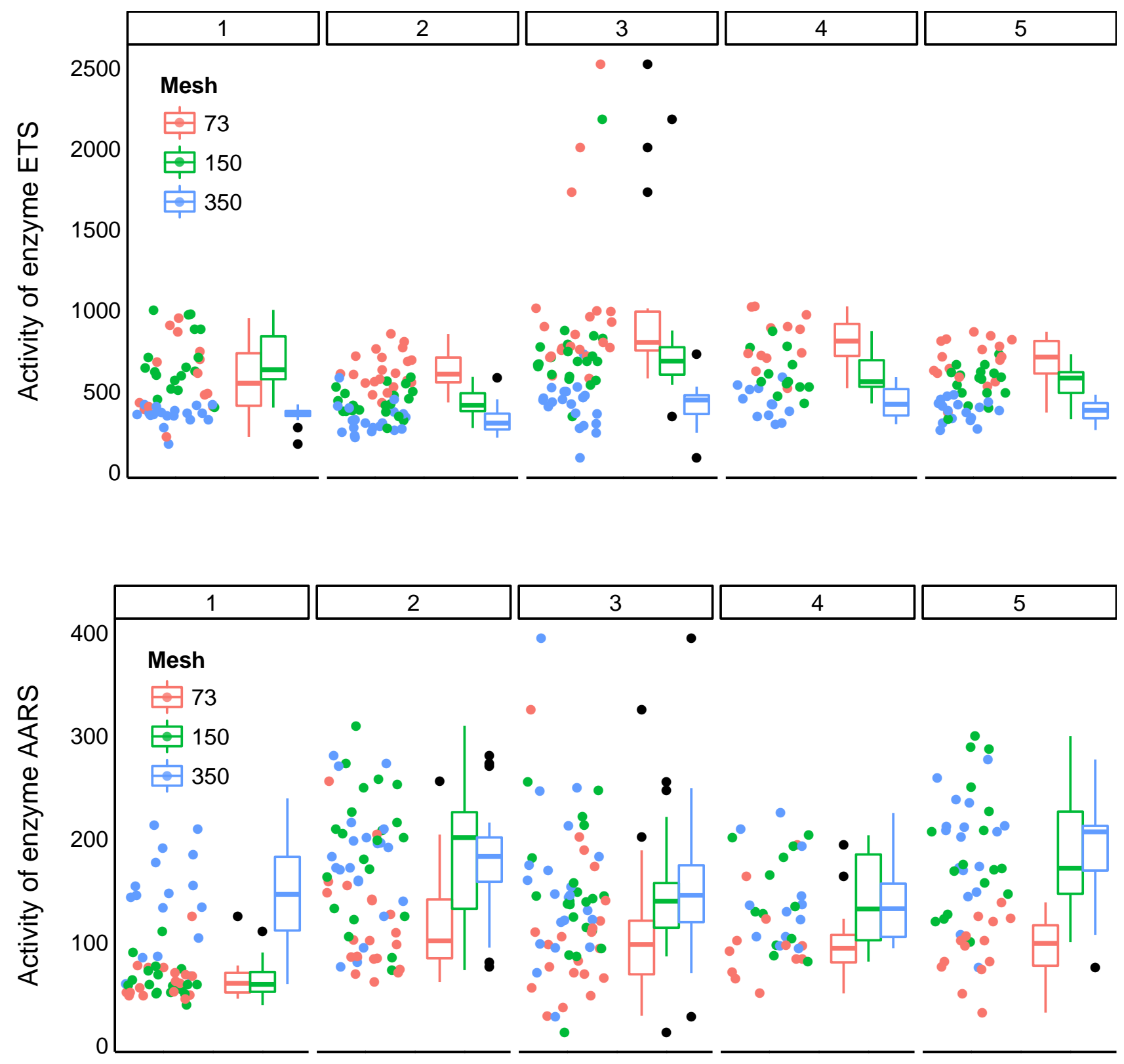

Figure B. plot of chunk eda 

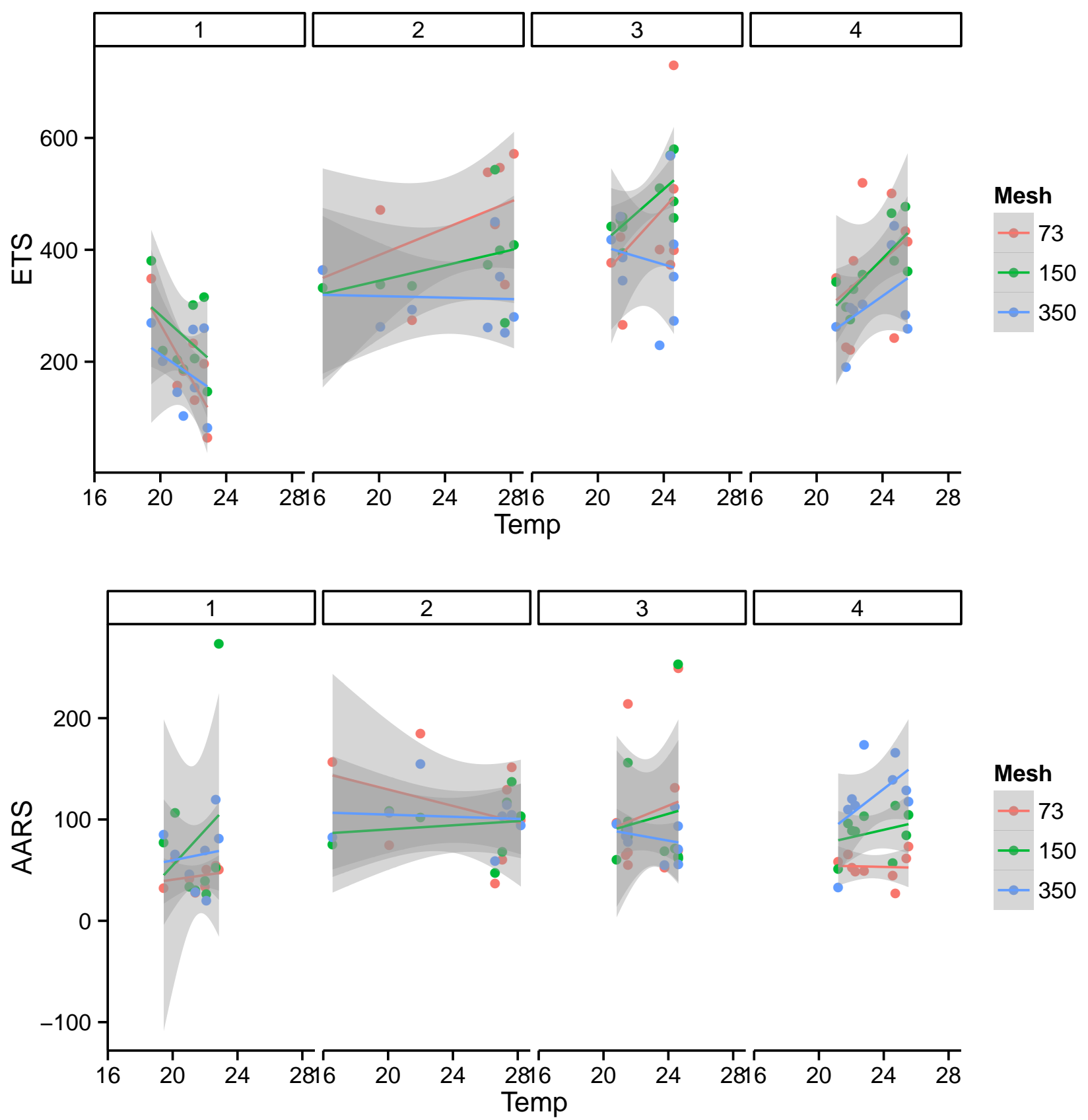

Figure C. plot of chunk eda1 

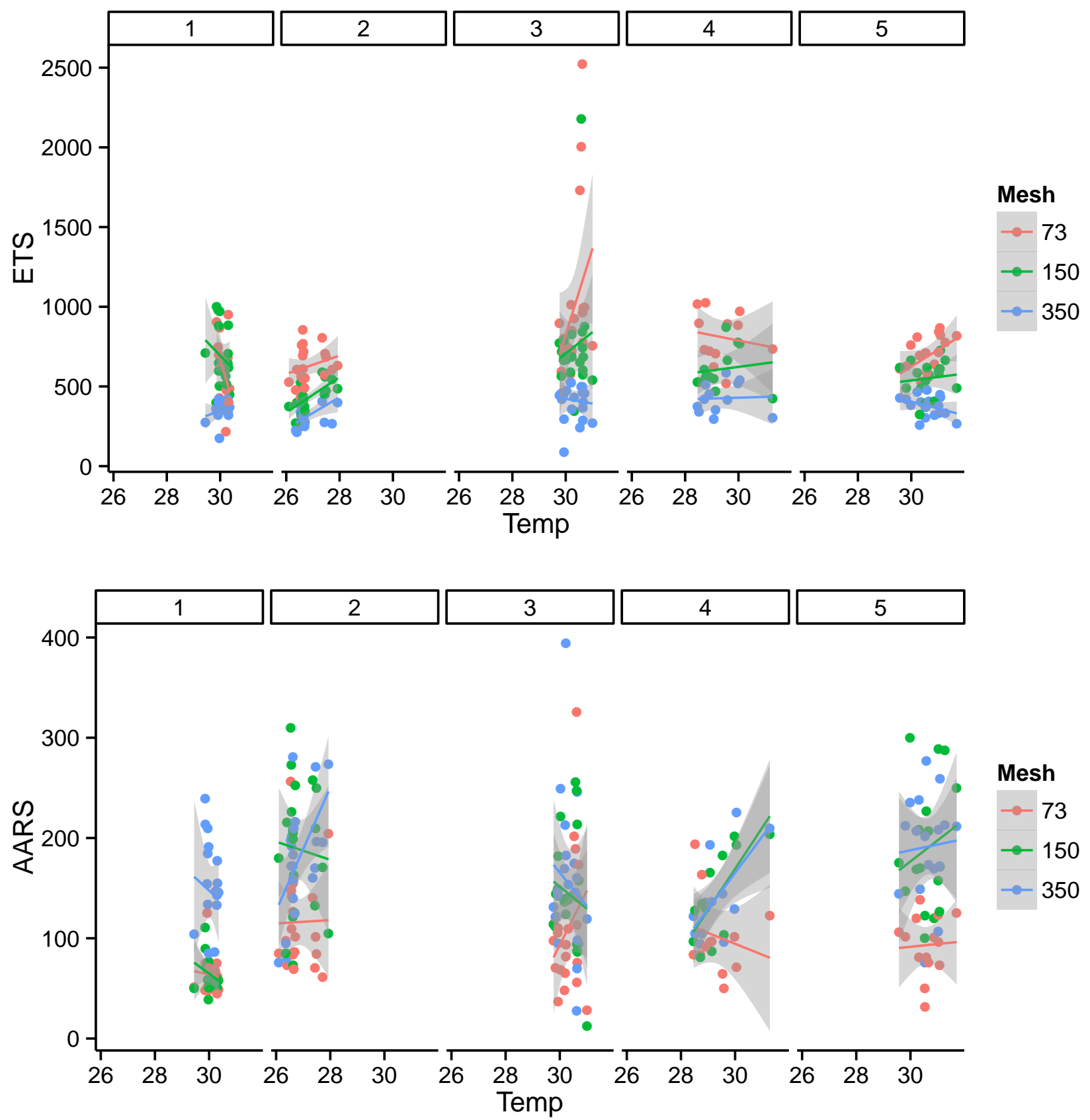

Figure D. plot of chunk eda1 


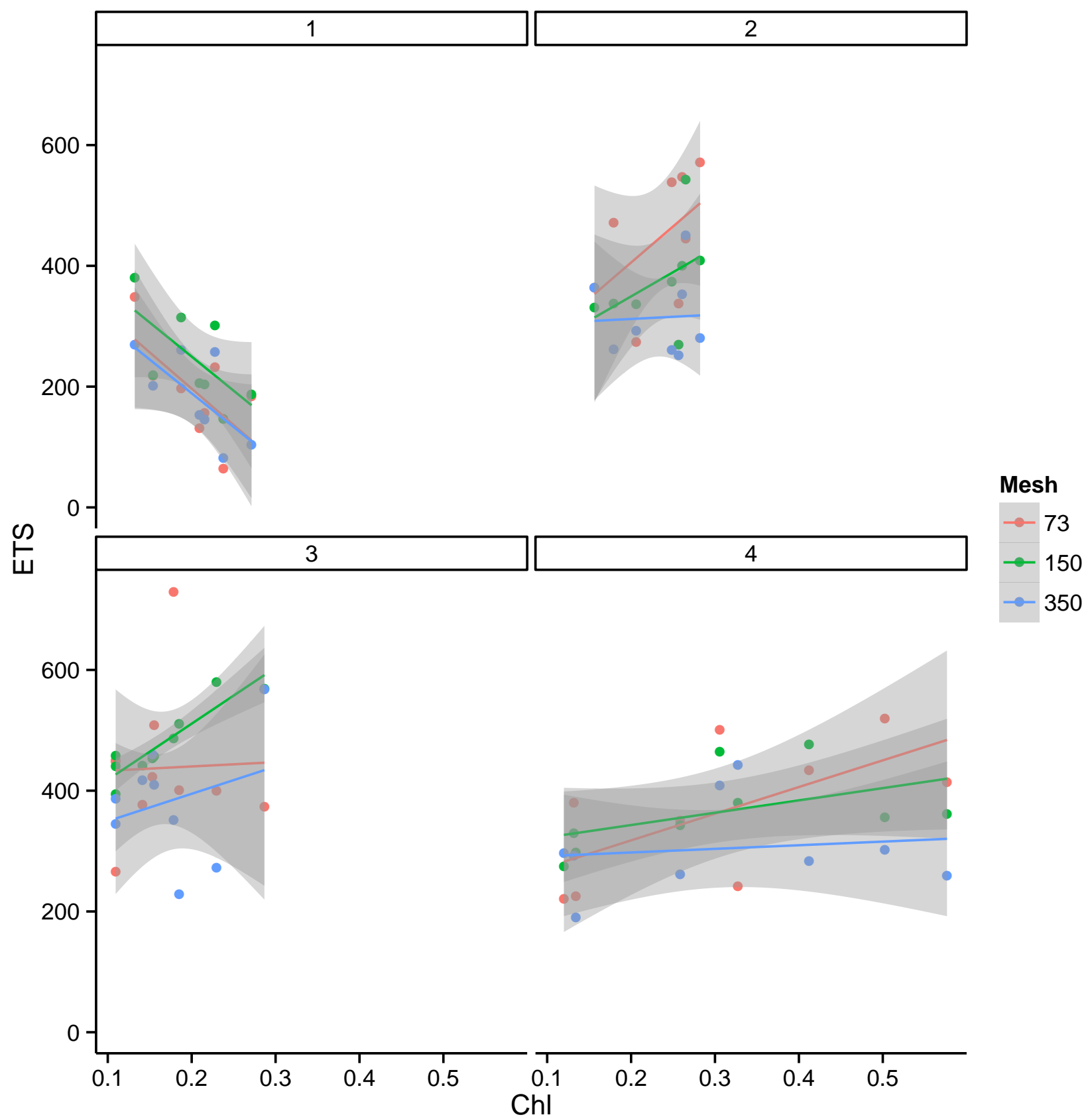

Figure E. plot of chunk eda2 


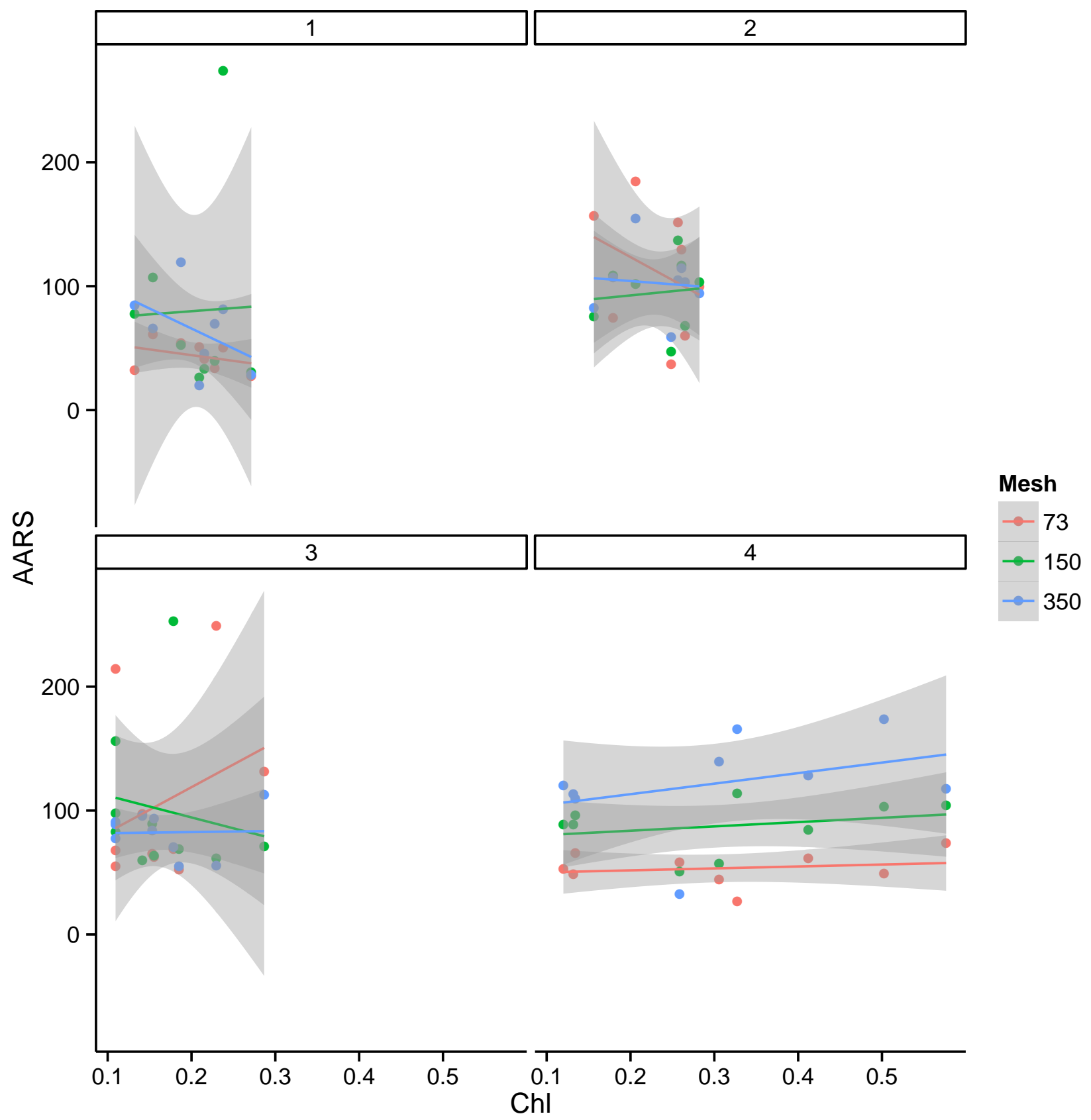

Figure F. plot of chunk eda2 


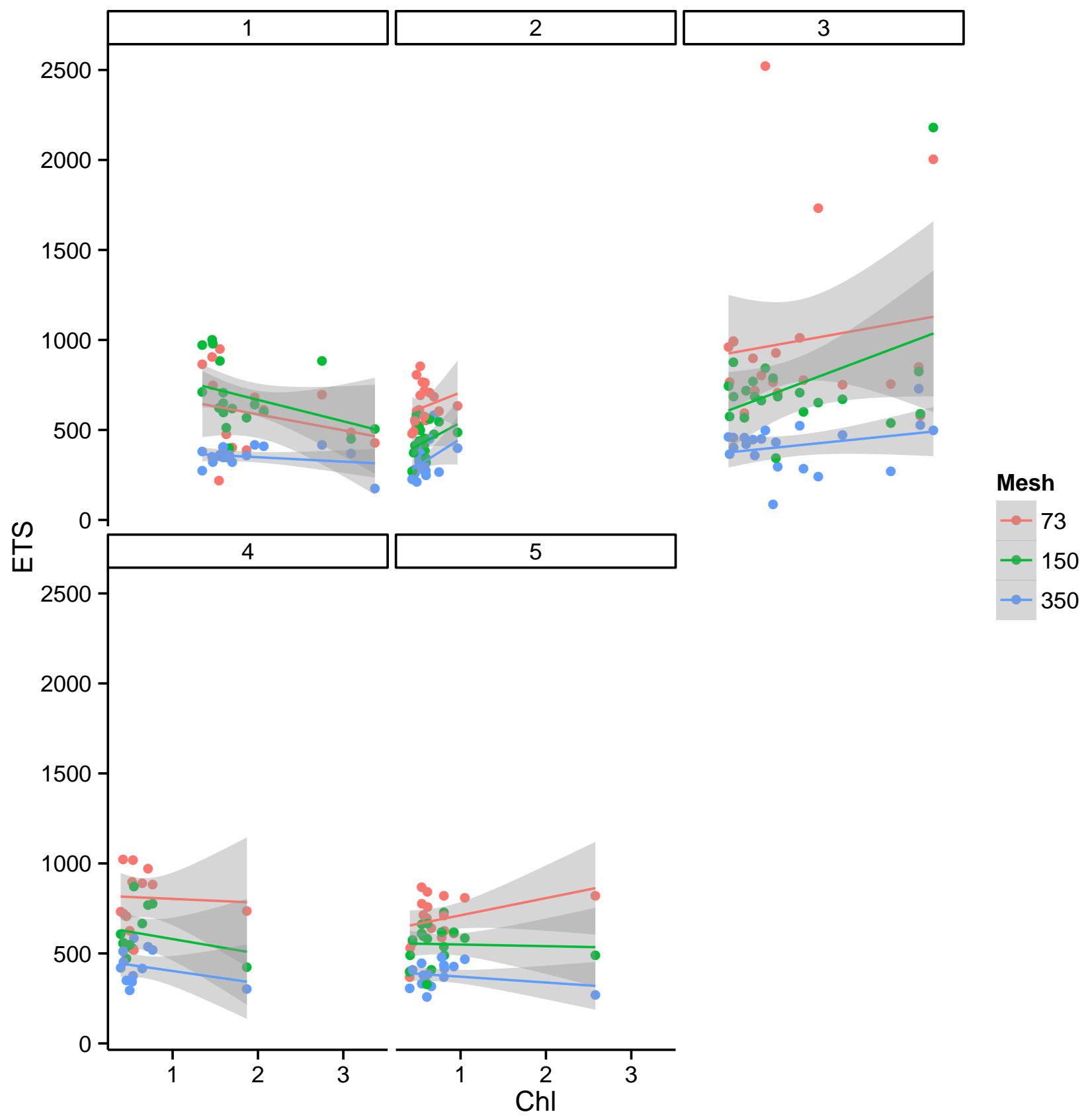

Figure G. plot of chunk eda2 


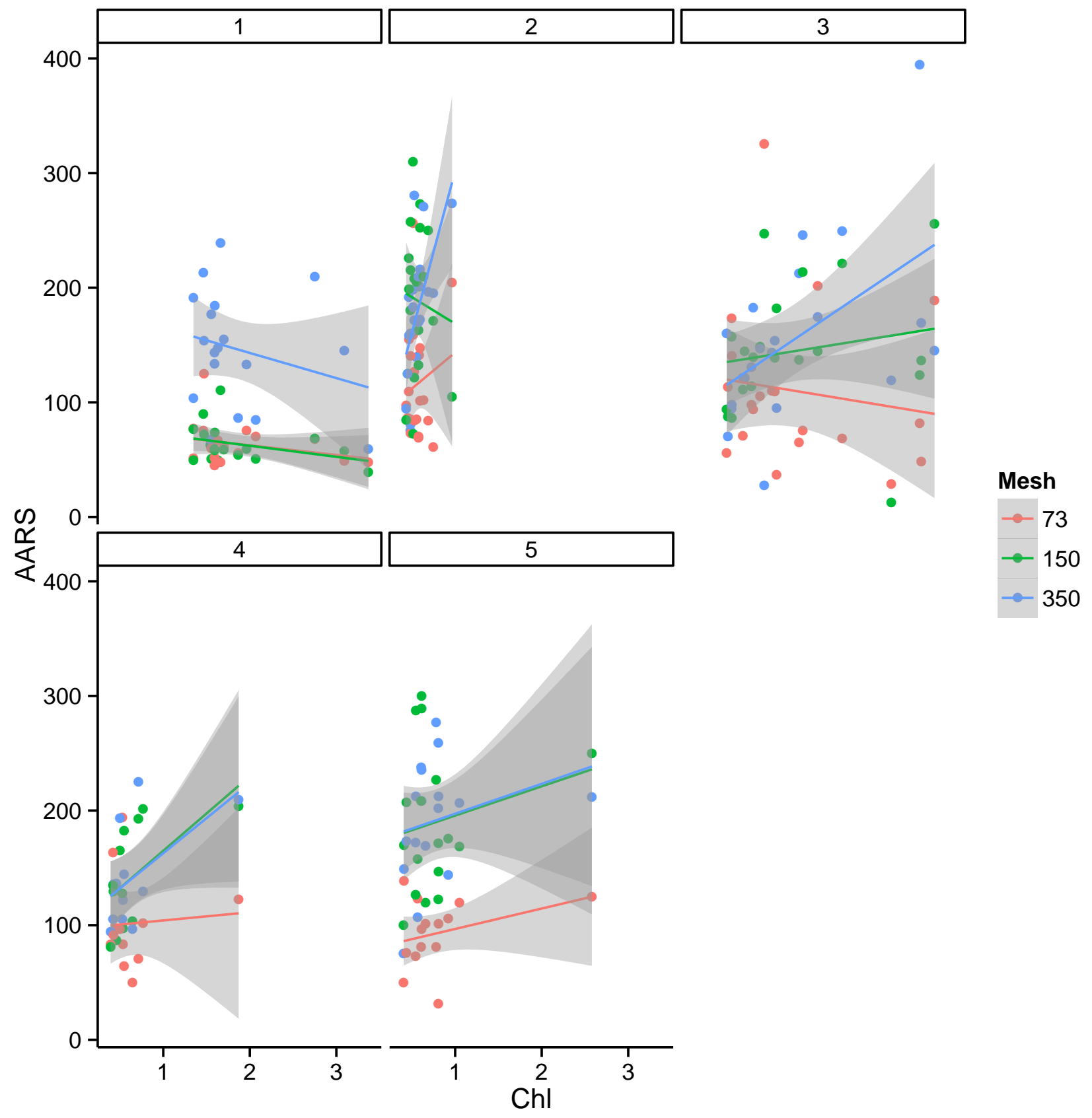

Figure $\mathbf{H}$. plot of chunk eda2 


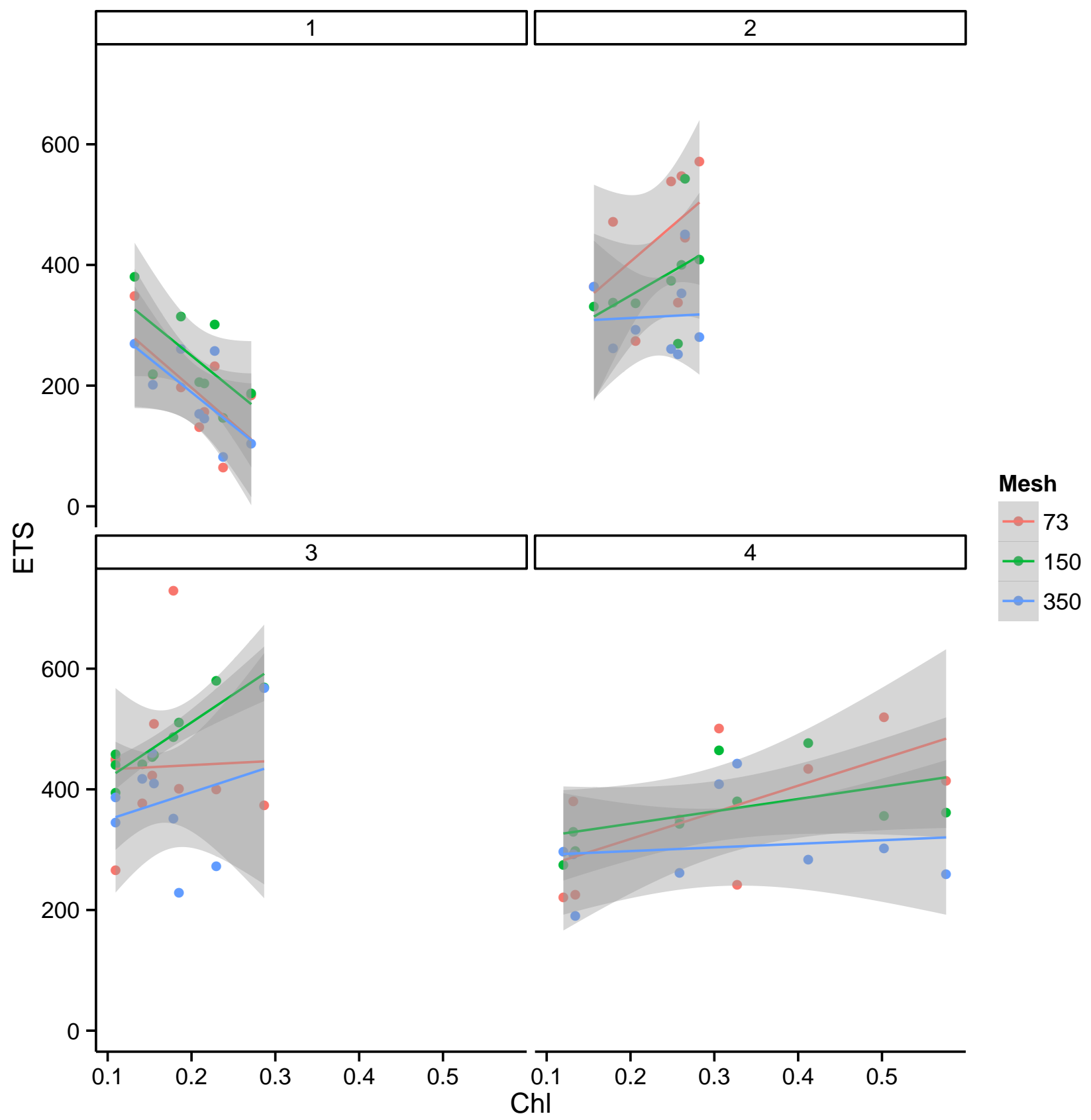

Figure I. plot of chunk eda3 


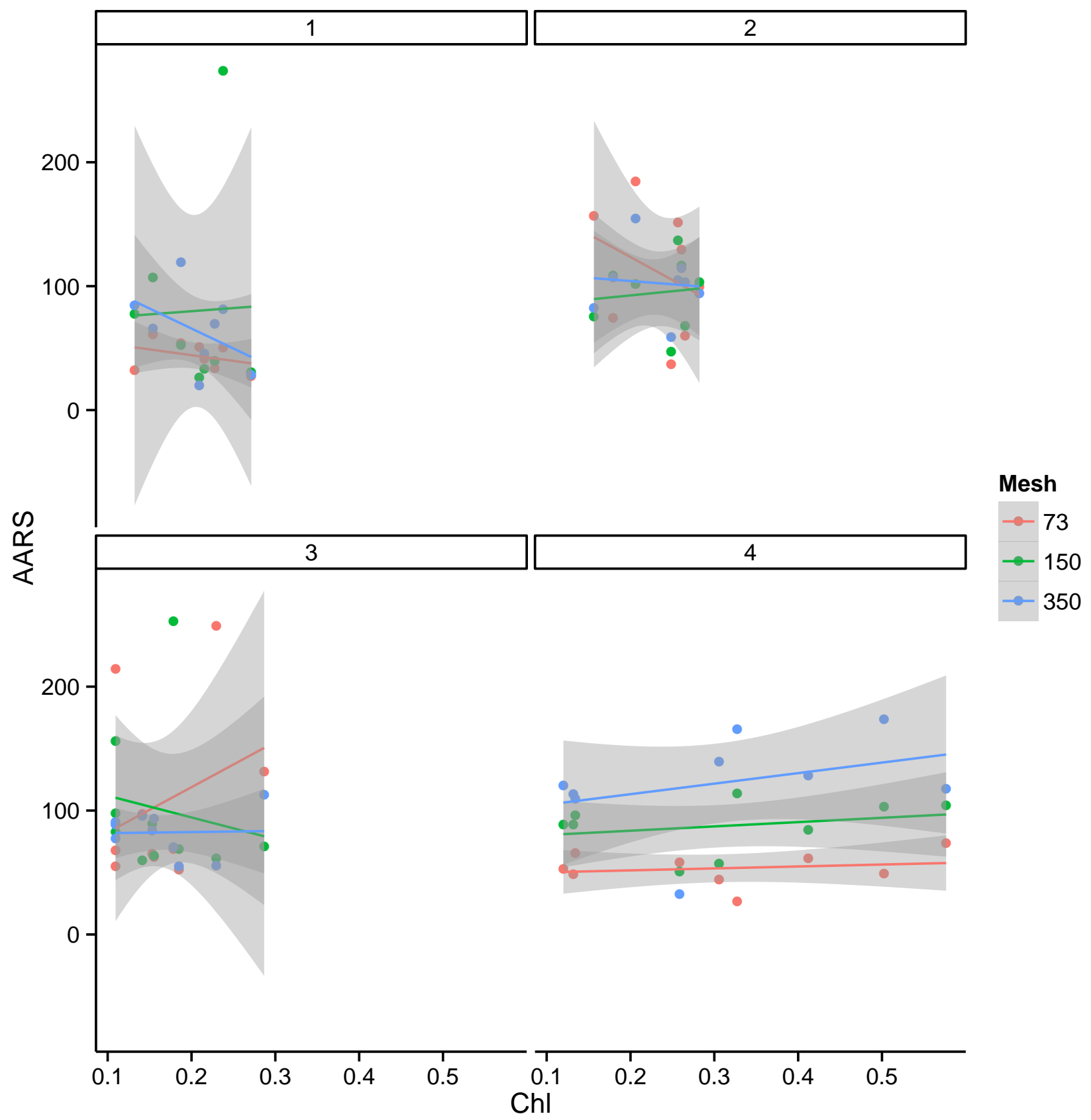

Figure J. plot of chunk eda3 


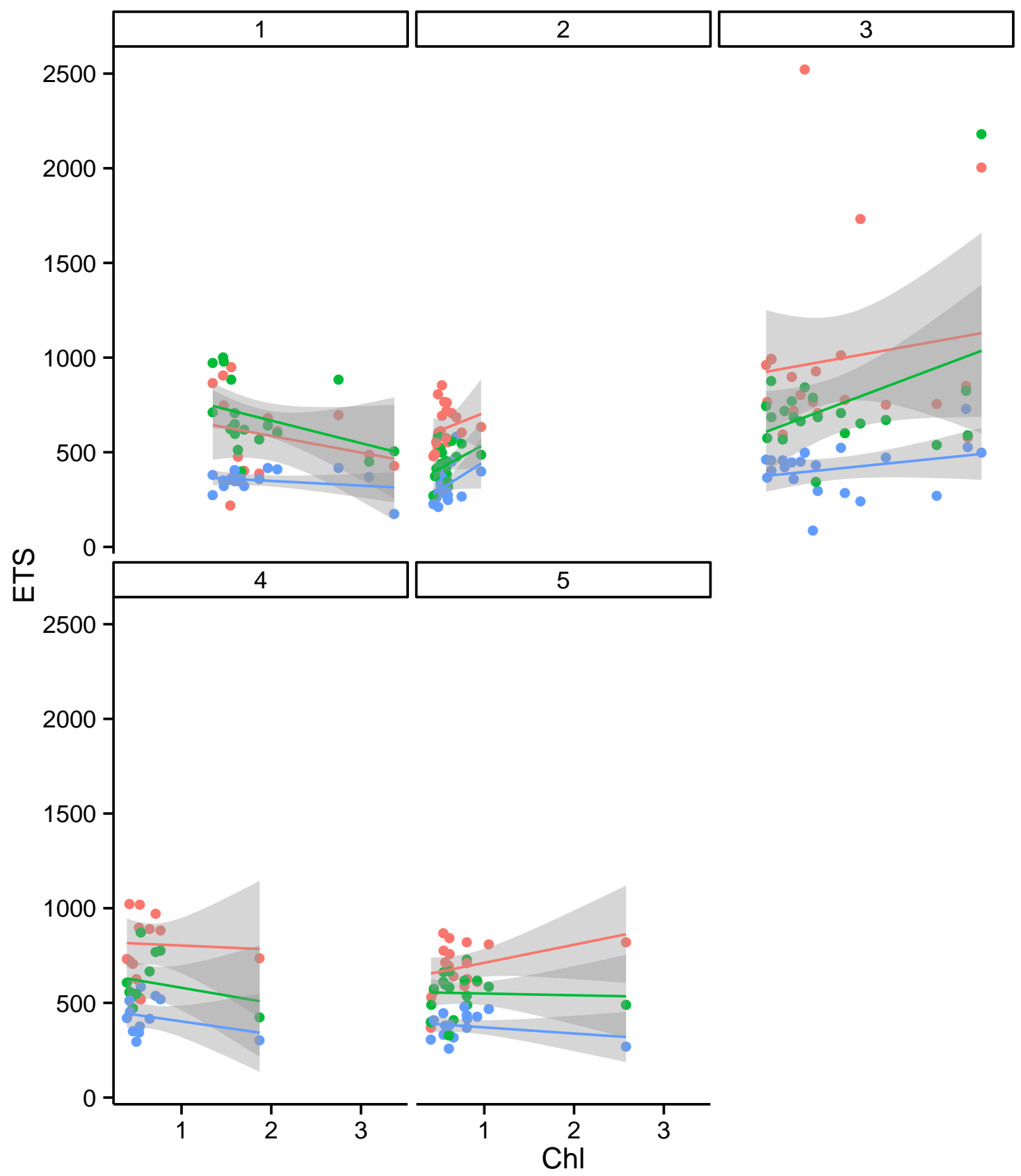

Mesh

$-\rightarrow 73$

$-150$

$-350$

Figure K. plot of chunk eda3 


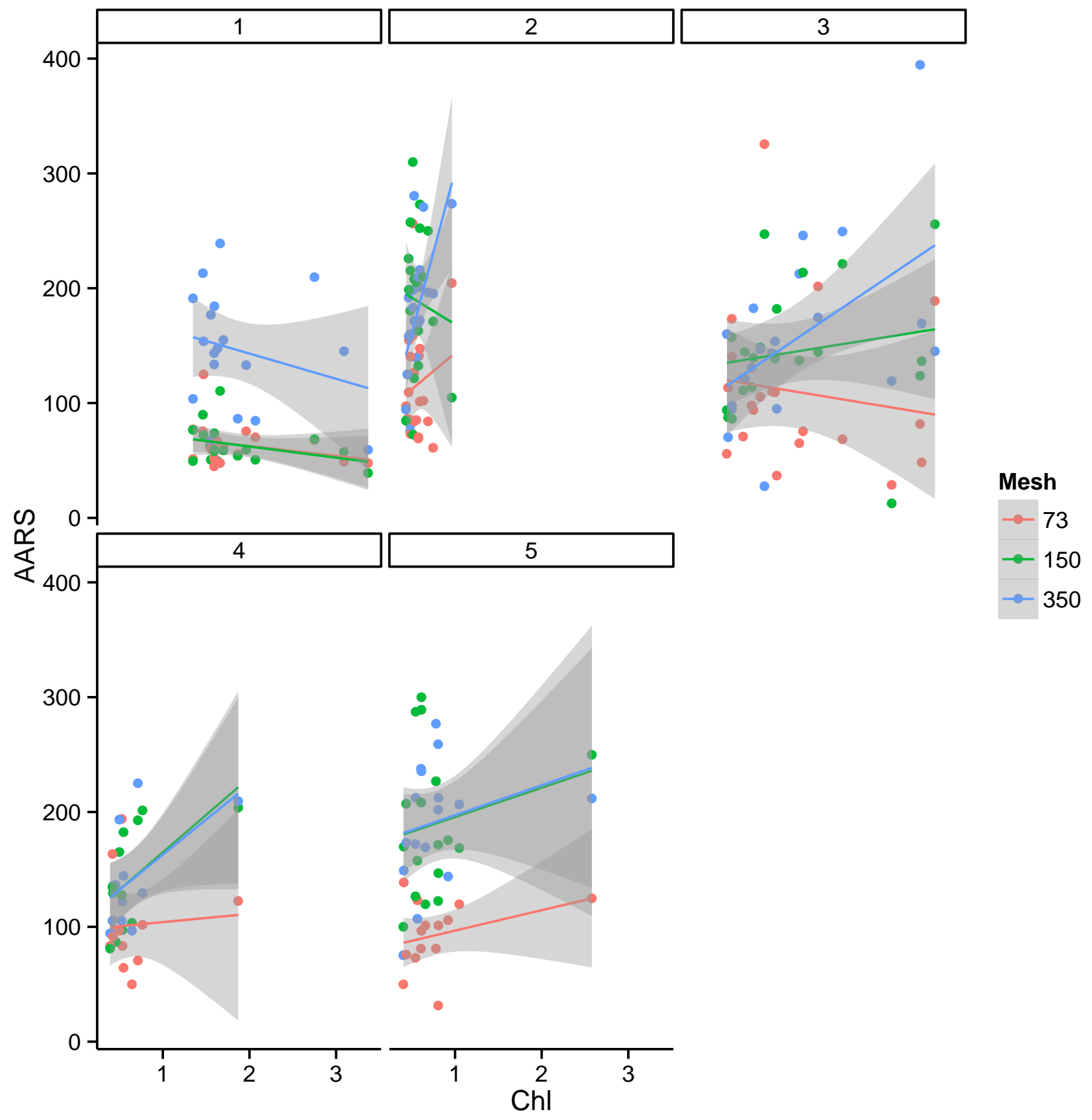

Figure L. plot of chunk eda3 
So what if we partial out protein, chl and temperature... If we want to estimate the effects of protein, chlorophyll and temperature, we first need to make sure that they are not correlated to one another (multi-collinearity). However, if we just wish to partial them out, then it is not so much of an issue.

library (GGally)

dat <- within(subset (csc, select=c(ETS, AARS, Protein, Chl, Temp)) , \{ $\log$ AARS $=\log ($ AARS)

logProtein=log (Protein)

+)

$\log C h l=\log (\mathrm{Chl})$

head(dat)

ETS AARS Protein Chl Temp logChl logProtein logAARS

$\begin{array}{llllllllll}63.79607 & 50.53627 & 0.17469550 & 0.2378516 & 22.85509 & -1.436109 & -1.744711 & 3.922691\end{array}$

$\begin{array}{lllllllll}156.96130 & 41.39708 & 0.28281610 & 0.2154798 & 21.02649 & -1.534888 & -1.262958 & 3.723210\end{array}$

$\begin{array}{lllllllll}3 & 183.69280 & 27.46457 & 0.08221237 & 0.2713337 & 21.40192 & -1.304406 & -2.498450 & 3.312897\end{array}$

$\begin{array}{lllllllll}4 & 232.62470 & 33.56256 & 0.25477210 & 0.2276602 & 21.99414 & -1.479901 & -1.367386 & 3.513411\end{array}$

$\begin{array}{lllllllll}5 & 217.68900 & 61.14242 & 0.05529095 & 0.1539380 & 20.15350 & -1.871205 & -2.895146 & 4.113206\end{array}$

$\begin{array}{lllllllll}6 & 348.08290 & 32.42767 & 0.09302974 & 0.1321699 & 19.44771 & -2.023667 & -2.374836 & 3.479012\end{array}$

p <- ggpairs (dat, columns $=c(c(1,8,5: 7))$,

lower=list (continuous="smooth", params=c $($ colour="blue" $)$ ),

diag=list (continuous="density", params=c ( colour="blue")),

upper $=1$ ist $($ params $=1$ ist $(\operatorname{corSize}=6))$, axisLabels $=$ ' show')

$>\operatorname{print}(\mathrm{p})$

I very much doubt that adding Temperature, Chlorophyll and Protein are going to do much... 


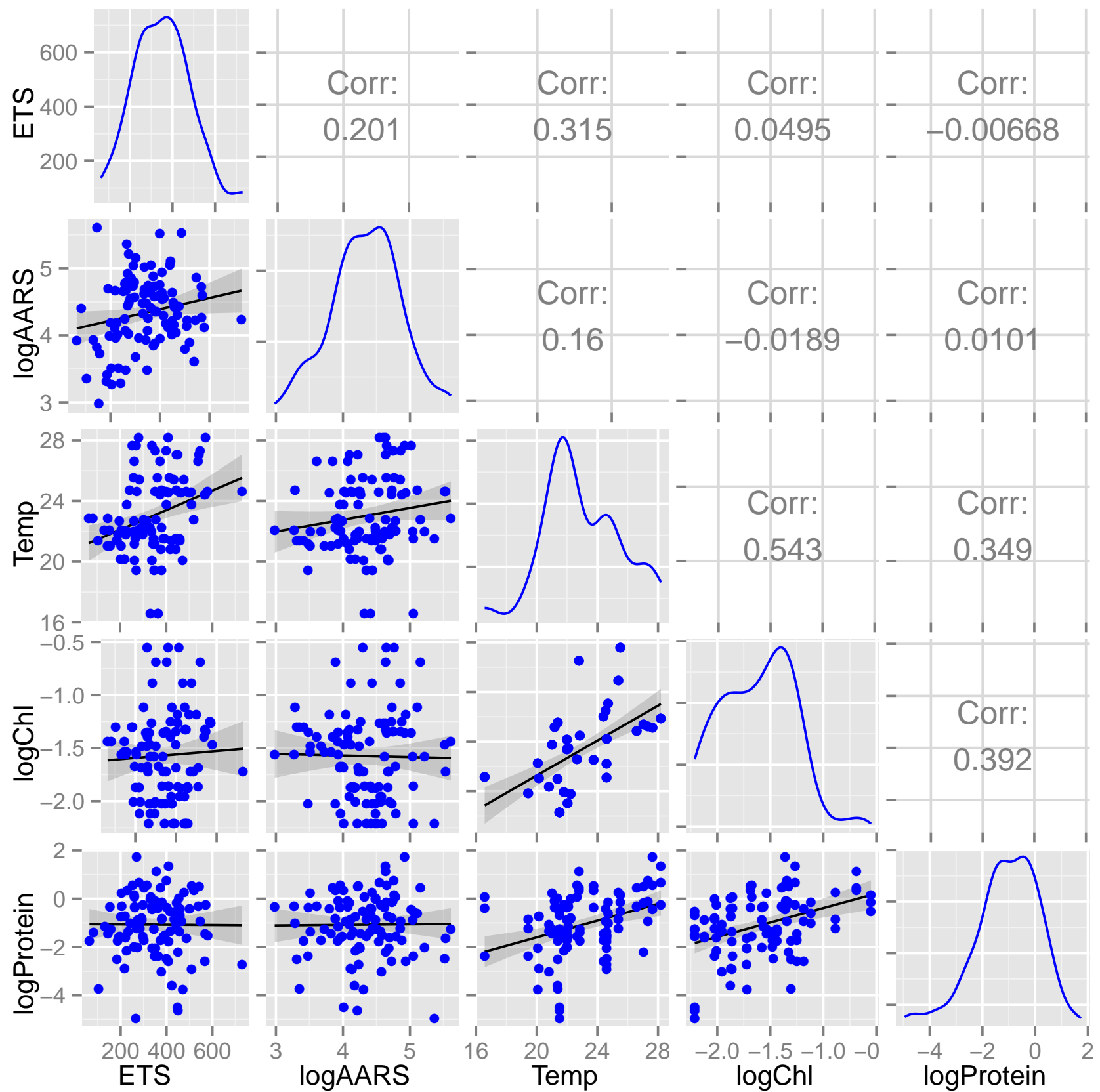

Figure M. plot of chunk eda4 


\section{Analyses}

For each location, the effects of mesh size, cruise (and their interactions) on ETS and AARS were explored via linear models in a Bayesian framework using JAGS (Plummer 2003) interfaced through R (R Core Team 2014) (Team 2014) and the R2jags (Su and Yajima 2014) package. Models also included the covariates of protein (log transformed), chlorophyll (log transformed) and Temperature in an attempt to reduce residual uncertainty.

$$
\begin{aligned}
y_{i} & \sim \mathcal{N}\left(\mu_{i}, \sigma^{2}\right) \\
\mu_{i} & =\beta_{0}+\beta X_{i}+\gamma W_{i} \\
X_{i j k} & =\text { mesh }_{i j}+\text { cruise }_{i k}+\text { mesh }: \text { cruise }_{i j k} \\
W_{i} & =\log \left(\text { protein }_{i}\right)+\log \left(\text { chlorophyll }_{i}\right)+\text { temperature }_{i} \\
\beta_{0} & \sim \mathcal{N}(0,100000) \\
\beta & \sim \mathcal{M V N}(0,1000000) \\
\gamma & \sim \mathcal{M V N}(0,1000000) \\
\sigma^{2} & \sim \mathcal{H C}(25)
\end{aligned}
$$

where $y_{i}$ are the $i_{\text {th }}$ ETS or AARS observation from the $j_{t h}$ mesh and $k_{\text {th }}$ cruise. Non-informative normal priors were specified for the intercept $\left(\beta_{0}\right)$ and non-informative multivariate normal priors were specified for both the main effect $(\beta)$ and covariate $(\gamma)$ parameters. Half-cauchy (scale $=25$ ) priors where specified for the variance following (Gelman 2006).

A total of 10,000 Gibbs sampling iterations were performed across 3 chains with a burnin of 1,000 and thinning rate of 10 , resulting in a total of 2,700 collected samples. Chain mixing and convergence were assessed via traceplots, autocorrelation and Gelman-Rubin diagnostics (all scale reduction factors less than 1.005)

The relative influences of the main effects of mesh, cruise and their interactions as well as the covariates ( protein, chlorophyll and temperature) were assessed via finite-population standard deviations (Gelman and Hill 2007). Pairwise comparisons of mesh within each cruise and vice verse were derived from specific contrasts on the posteriors and all inferences about specific differences (effects) were based on $95 \%$ Bayesian UIs for modeled higher posterior density (HPD) median effects.

\subsection{GBR}




\subsubsection{ETS}

$>\operatorname{model}={ }^{\prime}$

model \{

for ( $i$ in $1: n$ ) \{

$y[i] \sim \operatorname{dnorm}(\mathrm{mu}[i], \mathrm{tau})$

$\operatorname{mu}[i]<-\operatorname{inprod}(\operatorname{beta}[], X[i])+,i n p r o d(\operatorname{gamma}[], X X[i]$,

$$
\text { \} }
$$

beta dmnorm $(\mathrm{a} 0, \mathrm{AO})$

gamma dmnorm $(\mathrm{cO}, \mathrm{CO})$

tau <- pow (sigma, -2)

sigma $<-\mathrm{z} / \mathrm{sqrt}(\mathrm{chSq})$

$z \sim \operatorname{dnorm}(0,0.0016) I(0) \quad \# ,1 / 25 \sim 2=0.0016$

chSq dgamma $(0.5,0.5)$

sd.res <- sd $(y \cdot \operatorname{err}[])$

$\mathrm{b}[1]<-0$

b $[2: 3]<-\operatorname{beta}[2: 3]$

sd.mesh $<-$ sd $(b)$

$c[1]<-0$

$c[2: 4]<-\operatorname{beta}[4: 6]$

sd.cruise <- sd(c)

for ( $i$ in $1: 6$ ) $\{d[i]<-0\}$

$\mathrm{d}[7: 12]<-$ beta $[7: 12]$

sd.int $<-\operatorname{sd}(d)$

sd.protein <- abs (gamma [1])*sd $(X X[, 1])$

sd.chl <- abs (gamma [2])*sd $(X X[, 2])$

sd.temp <- abs (gamma [3])*sd(XX[, 3])

contrasts (csc $\$$ Mesh) <- contr.treatment

contrasts (csc\$Cruise) <- contr.treatment

dat1 <- subset(csc, !is.na(Protein))

$>\mathrm{X}<-$ model.matrix ( ${ }^{M e s h} *$ Cruise, data=dat1)

$>\mathrm{nX}<-\mathrm{ncol}(\mathrm{X})$

XX $<-$ model.matrix $(\sim \log ($ Protein $)+\log (\mathrm{Chl})+$ Temp, dat 1$)[,-1]$

$\mathrm{nXX}<-\mathrm{ncol}(\mathrm{XX})$

csc.list <- with(dat1, list $(y=E T S$,

n=nrow(dat1),

$\mathrm{X}=\mathrm{X}, \mathrm{nX}=\mathrm{nX}$,

$\mathrm{XX}=\mathrm{XX}$,

$\mathrm{a} 0=\operatorname{rep}(0, \mathrm{nX}), \mathrm{A} 0=\operatorname{diag}(1.0 \mathrm{E}-06, \mathrm{nX})$,

$\mathrm{c} 0=\operatorname{rep}(0, \mathrm{nXX}), \quad \mathrm{C} 0=\operatorname{diag}(1.0 \mathrm{E}-06, \mathrm{nXX})))$

param <- c('beta', 'gamma', 'sigma', 'sd.res', 'sd.mesh','sd.cruise', 'sd.int', 'sd.protein', 'sd.chl', 'sd.temp')

csc.jags <- jags (data=csc.list, init=NULL, parameters.to.save=param,

$$
\begin{aligned}
& \text { model } . \text { file=tex } \\
& \text { n. chains }=3 \text {, } \\
& \text { n. iter }=10000, \\
& \text { n. burnin }=1000, \\
& \text { n.thin=10) }
\end{aligned}
$$

Compiling model graph

Resolving undeclared variables

Allocating nodes

Graph Size: 2394

Initializing model

csc.jags

Inference for Bugs model at "5", fit using jags,

3 chains, each with 10000 iterations (first 1000 discarded), n.thin = 10

n.sims $=2700$ iterations saved

$\begin{array}{rrrrrrrrr}\text { mu.vect } & \text { sd.vect } & 2.5 \% & 25 \% & 50 \% & 75 \% & 97.5 \% & \text { Rhat } \text { n.eff } \\ 158.783 & 137.113 & -118.662 & 67.214 & 159.437 & 251.049 & 424.079 & 1.001 & 2700 \\ 58.188 & 44.008 & -28.529 & 28.570 & 58.229 & 86.950 & 143.654 & 1.000 & 2700 \\ -5.380 & 43.121 & -88.330 & -34.326 & -4.895 & 22.548 & 78.835 & 1.003 & 960 \\ 233.231 & 44.184 & 144.548 & 204.462 & 233.533 & 262.363 & 319.241 & 1.001 & 2700 \\ 242.935 & 44.188 & 152.640 & 214.401 & 244.027 & 271.950 & 326.218 & 1.001 & 2700 \\ 157.038 & 43.260 & 73.160 & 128.124 & 157.341 & 186.318 & 242.360 & 1.001 & 2700 \\ -118.560 & 62.134 & -240.526 & -158.773 & -119.914 & -77.973 & 3.581 & 1.001 & 2700\end{array}$




$\begin{array}{lrrrrrrrrr}\text { beta[8] } & -115.124 & 61.766 & -240.722 & -154.989 & -114.449 & -74.153 & 7.164 & 1.003 & 830 \\ \text { beta[9] } & -5.937 & 58.164 & -116.429 & -45.306 & -6.362 & 31.754 & 112.770 & 1.001 & 2700 \\ \text { beta[10] } & -27.268 & 60.131 & -146.750 & -67.034 & -27.472 & 12.506 & 88.952 & 1.001 & 2700 \\ \text { beta[11] } & -55.337 & 58.709 & -166.947 & -94.026 & -55.598 & -18.295 & 63.623 & 1.001 & 2700 \\ \text { beta[12] } & -56.102 & 59.638 & -175.323 & -94.756 & -57.434 & -16.243 & 64.524 & 1.003 & 890 \\ \text { gamma[1] } & -4.753 & 9.929 & -24.709 & -11.250 & -4.836 & 1.684 & 15.571 & 1.001 & 2000 \\ \text { gamma[2] } & 45.630 & 29.718 & -12.108 & 26.161 & 46.101 & 64.846 & 106.523 & 1.001 & 2700 \\ \text { gamma[3] } & 4.584 & 4.627 & -4.490 & 1.504 & 4.532 & 7.662 & 13.947 & 1.001 & 2700 \\ \text { sd.ch1 } & 19.673 & 11.217 & 1.089 & 11.182 & 19.209 & 26.968 & 44.301 & 1.001 & 2700 \\ \text { sd.cruise } & 115.224 & 17.740 & 78.823 & 103.786 & 115.393 & 126.964 & 149.785 & 1.001 & 2700 \\ \text { sd.int } & 56.941 & 16.726 & 27.419 & 45.037 & 55.745 & 67.361 & 92.643 & 1.003 & 780 \\ \text { sd.mesh } & 42.768 & 19.239 & 9.764 & 28.557 & 41.963 & 55.475 & 82.142 & 1.001 & 2700 \\ \text { sd.protein } & 11.159 & 8.411 & 0.430 & 4.536 & 9.366 & 15.889 & 32.048 & 1.001 & 2300 \\ \text { sd.res } & 86.621 & 2.522 & 82.752 & 84.764 & 86.278 & 88.088 & 92.381 & 1.001 & 2700 \\ \text { sd.temp } & 13.644 & 9.457 & 0.456 & 6.149 & 12.142 & 19.627 & 35.663 & 1.001 & 2700 \\ \text { sigma } & 86.971 & 6.716 & 75.624 & 82.321 & 86.454 & 90.961 & 101.815 & 1.001 & 2700 \\ \text { deviance } & 1200.645 & 6.071 & 1190.937 & 1196.186 & 1199.899 & 1204.101 & 1214.317 & 1.001 & 2700\end{array}$

For each parameter, $n$.eff is a crude measure of effective sample size, and Rhat is the potential scale reduction factor (at convergence, Rhat=1).

DIC info (using the rule, $\mathrm{pD}=\operatorname{var}($ deviance) $/ 2$ )

$\mathrm{pD}=18.4$ and $\mathrm{DIC}=1219.1$

DIC is an estimate of expected predictive error (lower deviance is better).

\#\# Finite-population standard deviations

X.mesh.var <-csc.jags\$BUGSoutput\$sims.list [['sd.mesh']]-2

X.protein.var <- csc.jags\$BUGSoutput\$sims.list[['sd.protein']] 2

X.chl.var <- csc.jags\$BUGSoutput\$sims.list [['sd.chl'] ] 2

X.temp.var <- csc.jags\$BUGSoutput\$sims.list [['sd.temp'] ] -2

X.cruise.var <- csc.jags\$BUGSoutput\$sims.list[['sd.cruise'] ]-2

X.int.var <- csc.jags\$BUGSoutput\$sims.list [['sd.int'] ] -2

R.var <- csc.jags\$BUGSoutput\$sims.list [['sd.res'] ] ${ }^{-2}$

R2.X<- (X.mesh.var + X.cruise.var + X.int.var + X.protein.var + X.chl.var + X.temp.var)/(X.mesh.var + X.cruise.var + X.int.var + X.protein.var + X.chl

$>$ R2.mesh<- ((X.mesh.var)/(X.mesh.var + X.cruise.var + X.int.var + X.protein.var + R.var))

R2.cruise<-((X.cruise.var)/(X.mesh.var + X.cruise.var + X.int.var + X.protein.var + X.chl.var + X.temp.var + R.var))

R2.int<-( X.int.var $) /(X . m e s h . v a r+X . c r u i s e . v a r+X . i n t . v a r+X . p r o t e i n . v a r+X . c h l . v a r+X . t e m p . v a r+R . v a r))$

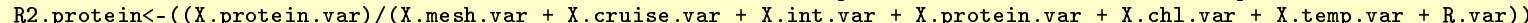

R2.chl<- ( X.chl.var)/(X.mesh.var + X.cruise.var + X.int.var + X.chl.var + X.chl.var + X.temp.var + R.var $))$

R2.temp<-((X.temp.var)/(X.mesh.var + X.cruise.var + X.int.var + X.temp.var + X.chl.var + X.temp.var + R.var ))

R2.resid <- ((R.var)/(X.mesh.var + X.cruise.var + X.int.var + X.protein.var + X.chl.var + X.temp.var + R.var))

a<-cbind(Residual=R.var, 'Temperature'=X.temp.var, 'Chlorophyll'=X.chl.var, 'Protein'=X.protein.var, 'Mesh $\mathrm{x}$ Cruise'=X.int.var, Cruise=X.cruise.var, sdtab <- cbind(Name=c ('Residuals', 'Temperature', 'Chlorophyll', 'Protein', 'Mesh x Cruise', 'Cruise', 'Mesh'), adply(a, 2, function(x) \{ data.frame $(\operatorname{Mean}=m e a n(x), \operatorname{Median}=\operatorname{median}(x)$, $\operatorname{HPDinterval}(\operatorname{as} \cdot \operatorname{mcmc}(\mathrm{x}))$, HPDinterval $(\operatorname{as} . \operatorname{mcmc}(\mathrm{x}), \mathrm{p}=0.68)$ )

\}))

a $<$-cbind(Residual=R2 .resid, 'Temperature'=R2.temp, 'Chlorophyll'=R2.chl, 'Protein'=R2 .protein, 'Mesh x Cruise'=R2.int, Cruise=R2 . cruise, Mesh=R2 .mesh R2tab <- cbind(Name=c('Residuals', 'Temperature', 'Chlorophyll', 'Protein', 'Mesh x Cruise', 'Cruise', 'Mesh'), adply(a, 2, function(x) \{ data.frame $(\operatorname{Mean}=\operatorname{mean}(\mathrm{x}), \operatorname{Median}=\operatorname{median}(\mathrm{x})$, $\operatorname{HPDinterval}(\operatorname{as} \cdot \operatorname{mcmc}(\mathrm{x}))$, $\operatorname{HPDinterval}(\operatorname{as} . \operatorname{mcmc}(\mathrm{x}), \mathrm{p}=0.68)$ ) \}))

sdtab\$R2 <- round $(\mathrm{R} 2$ tab\$Median, 3)

sdtab\$Name <- factor (sdtab\$Name, levels=c('Residuals', 'Temperature', 'Chlorophyll', 'Protein', 'Mesh x Cruise', 'Cruise', 'Mesh'))

sdtab <- subset $($ sdtab, select $=-\mathrm{X} 1)$

print(xtable(sdtab[7:1,], caption='Mean, median, (lower and upper) and highest probability density intervals of finite-population standard deviation \% latex table generated in R 3.1.0 by xtable 1.7-4 package \% Thu May 7 08:35:39 2015 
Table S1. Mean, median, (lower and upper) and highest probability density intervals of finite-population standard deviations as well as marginal $R^{2}$ approximations.

\begin{tabular}{lrrrrrrr}
\hline Name & Mean & Median & lower & upper & lower.1 & upper.1 & R2 \\
\hline Mesh & 2199.09 & 1760.93 & 4.54 & 5814.05 & 0.77 & 2634.47 & 0.07 \\
Cruise & 13591.25 & 13315.60 & 5649.63 & 21550.91 & 9200.58 & 17120.02 & 0.49 \\
Mesh x Cruise & 3521.91 & 3107.55 & 365.43 & 7662.14 & 1145.01 & 4541.36 & 0.12 \\
Protein & 195.24 & 87.71 & 0.00 & 743.45 & 0.00 & 189.28 & 0.00 \\
Chlorophyll & 512.82 & 368.99 & 0.00 & 1578.56 & 0.00 & 612.80 & 0.01 \\
Temperature & 275.56 & 147.42 & 0.00 & 984.12 & 0.00 & 300.94 & 0.01 \\
Residuals & 7509.60 & 7443.88 & 6813.96 & 8437.30 & 6961.77 & 7760.70 & 0.28 \\
\hline
\end{tabular}

p1 <- $\operatorname{ggplot}(\mathrm{sdtab}$, aes $(\mathrm{y}=$ Name, $\mathrm{x}=$ Median $))+$

geom_vline $($ xintercept $=0,1$ inetype='dashed' $)+$

geom_errorbarh (aes(xmin=lower, xmax=upper), height $=0)+$

geom_errorbarh (aes (xmin=lower.1, xmax=upper.1), height=0, size=2)+

geom_point ( $\operatorname{size}=3$, shape $=21$, fill=' white' $)+$

geom_text (aes (label=R2, $y=$ as .numeric (Name) +0.3), position=position_dodge (height=1), size $=3$ ) +

scale_x_continuous ('Finite-population standard deviation') +

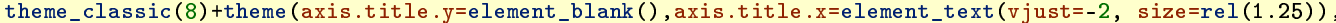
plot.margin=unit $(c(0,0,2,2)$, 'lines')

print (p1)

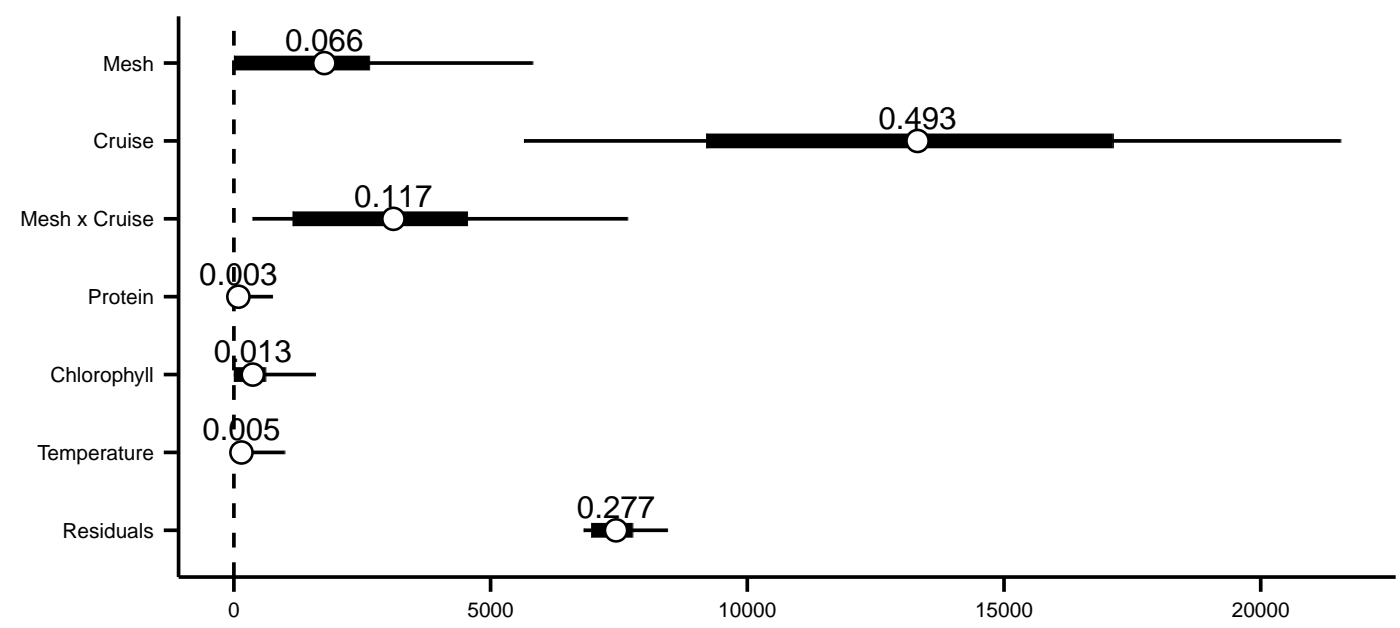

Finite-population standard deviation

Figure N. Median (lower and upper) highest probability density intervals of finite-population standard deviations. $R^{2}$ approximations above medians. 
newdata <- expand.grid(Mesh=levels (csc\$Mesh), Cruise=levels (csc\$Cruise))

$>$ newdata $\$$ Protein <- mean (csc\$Protein, na.rm=TRUE)

$>$ newdata $\$ C h l<-\operatorname{mean}(\csc \$ C h l$, na.rm=TRUE)

$>$ newdata\$Temp <- mean (csc\$Temp, na.rm=TRUE)

$>$ Xmat <- model.matrix ( Mesh*Cruise, data=newdata)

$>$ XXmat <- model.matrix ( $\log ($ Protein $)+\log (\mathrm{Chl})+$ Temp, data=newdata) $[,-1]$

$>$ coef <- csc.jags\$BUGSoutput\$sims.list [ ['beta']]

fit <- coef $\% * \%$ t(Xmat) + csc.jags $\$$ BUGSoutput $\$$ sims.list [['gamma'] ] \%*\% t (XXmat)

$>$ library (plyr)

$>$ newdata $<$ - cbind(newdata, adply(fit, 2, function(x) \{

data.frame $(\operatorname{Mean}=\operatorname{mean}(x), \operatorname{Median}=\operatorname{median}(x), \operatorname{HPDinterval}(\operatorname{as} . \operatorname{mcmc}(x)), \operatorname{HPDinterval}(\operatorname{as} . m c m c(x), p=0.68))$ \}))

$>$ \#newdata

\#Comparisons

MCMCsum <- function(x) \{

data.frame (Median=median ( $x$, na.rm=TRUE),

$t$ (quantile (x, na.rm=TRUE))

HPDinterval (as . mcmc ( $x$ ))

\} HPDinterval (as . mcmc (x), p=0.68))

library (multcomp)

$>$ ndata $<-$ newdata

$>$ ndata $<-$ subset (ndata, Cruise=='1')

Xmat <- contrMat (n=rep (1,nrow(ndata)), type="Tukey") \%*\% model.matrix ( Mesh*Cruise, ndata, xlev=list (Mesh=levels (csc $\$ M e s h)$, Cruise=levels (csc $\$$ Cruise)

$>$ wch <- ldply(strsplit (rownames (Xmat)," - "), as.numeric)

$>$ rownames (Xmat) <- paste (interaction(newdata\$Mesh, newdata $\$ C r u i s e)[w c h[, 1]], "-"$,interaction (newdata $\$$ Mesh, newdata $\$ C r u i s e)[w c h[, 2]])$

$>$ pairwise.comps <- coef $\% * \%$ t (Xmat)

$>$ comp.mesh_Cruise1<-adply (pairwise.comps, 2 , MCMCsum)

$>$ ndata $<-$ newdata

$>$ ndata $<-$ subset (ndata, Cruise=='2')

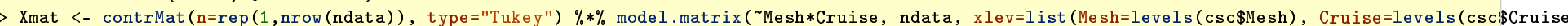

$>$ wch <- ldply(strsplit (rownames (Xmat)," - "), as .numeric)

$>$ rownames (Xmat) <- paste (interaction(newdata\$esh, newdata\$Cruise) [wch [,1]], "-", interaction(newdata $\$$ Mesh, newdata $\$ C r u i s e)[w c h[, 2]]$ )

$>$ pairwise.comps <- coef $\% * \%$ t (Xmat)

$>$ comp.mesh_Cruise2<-adply (pairwise.comps,2, MCMCsum)

ndata $<-$ newdata

ndata $<-$ subset (ndata, Cruise==' $\left.3^{\prime}\right)$

Xmat <- contrMat (n=rep (1,nrow (ndata)), type="Tukey") \%*\% model.matrix( ${ }^{M e s h} *$ Cruise, ndata, xlev=list (Mesh=levels (csc $\$$ Mesh), Cruise=levels (csc $\left.\$ C r u i s e\right)$

$>$ wch <- ldply(strsplit (rownames (Xmat)," - "), as.numeric)

$>\operatorname{rownames}($ Xmat) <- paste (interaction(newdata $\$$ Mesh, newdata $\$$ Cruise) [wch [,1]], "-", interaction(newdata $\$$ Mesh, newdata $\$ C r u i s e)[w c h[, 2]]$ )

pairwise.comps <- coef $\% * \%$ t (Xmat)

comp.mesh_Cruise3<-adply (pairwise.comps, 2 , MCMCsum)

ndata $<-$ newdata

ndata $<-$ subset (ndata, Cruise==' 4')

Xmat <- $\operatorname{contrMat(n=rep(1,nrow(ndata)),~type="Tukey")~\% *\% ~model.matrix~(~Mesh*Cruise,~ndata,~xlev=list~(Mesh=levels~(csc~} \$$ Mesh), Cruise=levels (csc $\$ C r u i s e)$

$>$ wch <- ldply(strsplit (rownames(Xmat)," - "), as.numeric)

rownames (Xmat) <- paste(interaction(newdata\$Mesh, newdata $\$ C r u i s e)[w c h[, 1]], "-"$, interaction (newdata $\$$ Mesh, newdata $\$ C r u i s e)[w c h[, 2]]$ )

pairwise.comps <- coef $\% * \%$ t (Xmat)

comp.mesh_Cruise4<-adply (pairwise.comps, 2, MCMCsum)

comp.mesh <- rbind(data.frame (Cruise=1, comp.mesh_Cruise1) data frame (Cruise $=2$, comp.mesh Cruise2) data.frame (Cruise=3, comp. mesh_Cruise 3 ) data.frame (Cruise $=4$, comp.mesh_Cruise4))

p1 <- ggplot (comp.mesh, aes ( $\mathrm{x}=$ Cruise, $\mathrm{y}=$ Median, color $=\mathrm{X} 1))+$ geom_hline (yintercept $=0$, linetype $=$ 'dashed') + geom_errorbar (aes (ymin=lower, ymax=upper), width=0, position=position_dodge (width=0.5)) + geom_errorbar (aes (ymin=lower.1, ymax=upper.1), width=0, size=2, position=position_dodge $($ width=0.5) $)+$ geom_point (size=3, shape=21, fill='white',position=position_dodge $($ width=0.5) $)$ +

scale_y_continuous('Median effect size') +

\#scale $x$ discrete ('Cruise number') t

scale_color_manual ('Mesh contrasts', breaks=c('150.1 - 73.1', '350.1 - 73.1','350.1 - 150.1'), labels=c('150 vs 73', coord_flip ()+theme_classic ()$+$

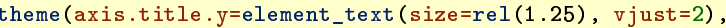

axis.title. $x=e l e m e n t$ text ( $\operatorname{size}=r e l(1.25)$, vjust $=-1)$,

plot . margin=unit $(c(0,0,1,1)$, 'lines' $))$

ndata <- newdata

ndata $<-$ subset (ndata, Mesh==' $73^{\prime}$ )

Xmat <- contrMat (n=rep (1, nrow (ndata)), type="Tukey") \%*\% model.matrix ( Mesh*Cruise, ndata, xlev=list (Mesh=levels (csc $\$$ Mesh), Cruise=levels (csc $\$$ Cruise) pairwise.comps <- coef $\% * \%$ t (Xmat)

comp.cruise_Mesh73<-adply (pairwise.comps,2, MCMCsum)

ndata $<-$ newdata

ndata<- $\operatorname{subset}\left(\right.$ ndata, Mesh==' $\left.150^{\prime}\right)$

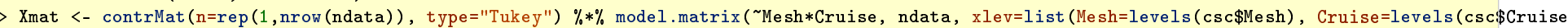

pairwise.comps <- coef $\% * \%$ t (Xmat)

comp.cruise_Mesh150<-adply(pairwise.comps,2, MCMCsum)

ndata $<-$ newdata

ndata<- subset (ndata, Mesh=='350')

Xmat <- contrMat (n=rep (1, nrow (ndata)), type="Tukey") \%*\% model.matrix ( Mesh*Cruise, ndata, xlev=list (Mesh=levels (csc $\$$ Mesh), Cruise=levels (csc $\$$ Cruise) pairwise.comps <- coef $\% * \%$ t (Xmat)

comp.cruise_Mesh350<-adply (pairwise.comps, 2, MCMCsum)

comp.cruise <- rbind(data.frame(Mesh=73, comp.cruise_Mesh73)

data.frame (Mesh=150, comp.cruise_Mesh150) data.frame (Mesh=350, comp. cruise_Mesh350)) 

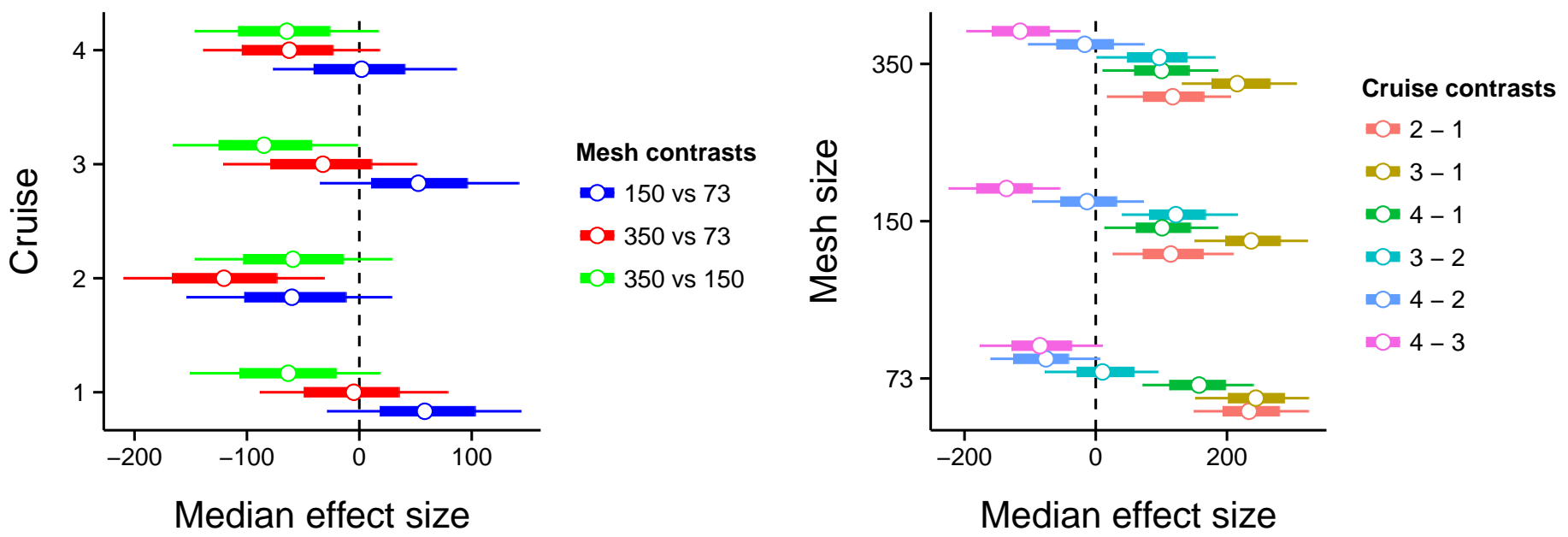

Figure O. plot of chunk ETScontrasts 


\subsubsection{AARS}

$>\operatorname{model}={ }^{\prime}$

model \{

for ( $i$ in $1: n)\{$

$y[i] \sim \operatorname{dnorm}(\mathrm{mu}[i], \mathrm{tau})$

$\mathrm{mu}[\mathrm{i}]<-\operatorname{inprod}(\operatorname{beta}[], \mathrm{X}[\mathrm{i}])+,\mathrm{inprod}(\operatorname{gamma}[], \mathrm{XX}[\mathrm{i}]$,

\}

$y \cdot \operatorname{err}[i]<-y[i]-\operatorname{mu}[i]$

beta dmnorm $(\mathrm{a} 0, \mathrm{AO})$

gamma dmnorm $(\mathrm{cO}, \mathrm{CO})$

tau <- pow (sigma, -2)

sigma $<-\mathrm{z} / \mathrm{sqrt}(\mathrm{chSq})$

$z \sim \operatorname{dnorm}(0,0.0016) \mathrm{I}(0) \quad \# ,1 / 25^{-2}=0.0016$

$\mathrm{chSq} \sim \operatorname{dgamma}(0.5,0.5)$

sd.res <- $\operatorname{sd}(\mathrm{y} \cdot \operatorname{err}[])$

$\mathrm{b}[1]<-0$

$\mathrm{b}[2: 3]<-\operatorname{beta}[2: 3]$

sd.mesh $<-\operatorname{sd}(b)$

$c[1]<-0$

$c[2: 4]<-\operatorname{beta}[4: 6]$

sd.cruise $<-$ sd $(c)$

for ( $i$ in $1: 6) \quad$ d [i] $<-0\}$

$d[7: 12]<-$ beta $[7: 12]$

sd.int $<-\operatorname{sd}(d)$

sd.protein <- $\operatorname{abs}(\operatorname{gamma}[1]) * \operatorname{sd}(\mathrm{XX}[, 1])$

sd.chl $<-$ abs $($ gamma $[2]) * \operatorname{sd}(\mathrm{XX}[, 2])$

\}

sd.temp <- abs (gamma [3])*sd $(X X[, 3])$

$>$ contrasts $(\csc \$$ Mesh $)<-$ contr.treatment

$>$ contrasts (csc\$Cruise) <- contr.treatment

dat1 <- subset(csc, ! is.na(Protein))

$>\mathrm{X}<-$ model.matrix ( Mesh $*$ Cruise, data=dat1)

$>\mathrm{nX}<-\mathrm{ncol}(\mathrm{X})$

$>\mathrm{XX}<-$ model.matrix $(\sim \log (\operatorname{Prote} i n)+\log (\mathrm{Ch} 1)+\operatorname{Temp}, \operatorname{dat} 1)[,-1]$

$\mathrm{nXX}<-\mathrm{ncol}(\mathrm{XX})$

$>$ csc.list <- with(dat1, list (y=AARS,

$+\quad n=$ nrow(dat1),

$\mathrm{X}=\mathrm{X}, \mathrm{nX}=\mathrm{nX}$

$\mathrm{XX}=\mathrm{XX}$,

$\mathrm{a} 0=\operatorname{rep}(0, \mathrm{nX}), \mathrm{A} 0=\operatorname{diag}(1.0 \mathrm{E}-06, \mathrm{nX})$,

$\mathrm{c} 0=\operatorname{rep}(0, \mathrm{nXX}), \mathrm{C} 0=\operatorname{diag}(1.0 \mathrm{E}-06, \mathrm{nXX})))$

$>$ param <- c('beta', 'gamma', 'sigma', 'sd.res', 'sd.mesh', 'sd.cruise', 'sd.int', 'sd.protein', 'sd.chl', 'sd.temp')

csc.jags <- jags (data=csc.list, init=NULL, parameters. to . save=param,

$$
\begin{aligned}
& \text { model } . \text { file }=\text { tex } \\
& \text { n. } \text { chains }=3 \text {, } \\
& \text { n. iter }=10000, \\
& \text { n. burnin }=1000,
\end{aligned}
$$

n.thin=10)

Compiling model graph

Resolving undeclared variables

Allocating nodes

Graph Size: 2394

Initializing model

csc.jags

\begin{tabular}{|c|c|c|c|c|c|c|c|c|}
\hline mu. vect & sd. vect & $2.5 \%$ & $25 \%$ & $50 \%$ & $75 \%$ & $97.5 \%$ & Rhat & $n \cdot \operatorname{eff}$ \\
\hline 25.049 & 72.736 & -109.549 & -25.097 & 24.395 & 74.175 & 169.432 & 1.001 & 2700 \\
\hline 41.000 & 24.051 & -5.369 & 24.783 & 41.019 & 57.030 & 88.743 & 1.001 & 270 \\
\hline 22.382 & 22.958 & -22.515 & 7.152 & 22.352 & 37.874 & 67.966 & 1.001 & 270 \\
\hline 66.763 & 23.725 & 20.329 & 50.527 & 66.829 & 82.325 & 114.001 & 1.001 & \\
\hline 56.153 & 23.709 & 8.460 & 40.582 & 56.134 & 71.811 & 102.268 & 1.002 & 110 \\
\hline 10.989 & 23.118 & -34.394 & -4.898 & 10.577 & 26.957 & 54.869 & 1.001 & 270 \\
\hline-49.043 & 33.238 & -113.765 & -71.587 & -48.699 & -26.996 & 16.986 & 1.001 & 270 \\
\hline
\end{tabular}

Inference for Bugs model at "5", fit using jags,

3 chains, each with 10000 iterations (first 1000 discarded), n.thin = 10

$\mathrm{n} . \mathrm{sims}=2700$ iterations saved 


\begin{tabular}{|c|c|c|c|c|c|c|c|c|c|}
\hline veta [8] & -28.224 & 33.369 & -92.057 & -50.727 & -28.788 & -5.915 & 37.989 & 1.001 & 2700 \\
\hline beta [9] & -35.291 & 31.380 & -96.039 & -57.027 & -35.117 & -14.708 & 27.055 & 1.001 & 700 \\
\hline eta $[10]$ & -34.409 & 31.981 & -95.847 & -56.496 & -34.915 & -12.666 & 30.913 & 1.000 & 700 \\
\hline eta [11] & -2.786 & 32.269 & -66.741 & -24.261 & -3.274 & 18.649 & 59.278 & 1.001 & \\
\hline eta [12] & 46.364 & 32.017 & -17.438 & 25.223 & 46.608 & 67.625 & 110.122 & 1.001 & 10 \\
\hline amma [1] & -5.303 & 5.295 & -15.343 & -8.984 & -5.308 & -1.722 & 5.116 & 1.001 & \\
\hline amma [2] & 6.191 & 15.478 & -23.603 & -3.959 & 5.940 & 16.139 & 36.828 & 1.001 & \\
\hline amma [3] & 0.905 & 2.472 & -4.128 & -0.793 & 0.993 & 2.619 & 5.673 & 1.001 & 270 \\
\hline d.chl & 5.465 & 4.264 & 0.236 & 2.085 & 4.628 & 7.686 & 34 & 1.003 & \\
\hline d.cruise & 35.713 & 9.357 & 17.300 & 29.490 & 35.723 & 41.919 & 54.096 & 1.001 & 240 \\
\hline d.int & 31.441 & 8.079 & 16.641 & 25.833 & 30.911 & 36.751 & 47 & 1.001 & \\
\hline d.mesh & 24.179 & 10.589 & 5.666 & 16.383 & 23.719 & 31.321 & 46.464 & 1.002 & $27 c$ \\
\hline d.protein & 7.851 & 5.372 & 0.375 & 3.478 & 7.098 & 11.510 & 19.479 & 1.001 & 270 \\
\hline d.res & 45.645 & 1.305 & 43.674 & 44.672 & 45.468 & 46.394 & 48.644 & 1.001 & 270 \\
\hline sd.temp & 5.411 & 3.967 & 0.220 & 2.326 & 4.629 & 7.781 & 14.997 & 1.001 & 270 \\
\hline i. & 45.874 & 3.469 & 39.634 & 43.467 & 45.699 & 48.087 & 53.339 & 1.002 & 180 \\
\hline evis & 1080.290 & 6.186 & 1070.653 & 1075.793 & 1079.588 & 1083.926 & 1094.069 & 1.000 & \\
\hline
\end{tabular}

For each parameter, $n$.eff is a crude measure of effective sample size, and Rhat is the potential scale reduction factor (at convergence, Rhat=1).

DIC info (using the rule, $\mathrm{pD}=\operatorname{var}($ deviance) $/ 2$ )

$\mathrm{pD}=19.1$ and $\mathrm{DIC}=1099.4$

DIC is an estimate of expected predictive error (lower deviance is better).

\#\# Finite-population standard deviations

$\mathrm{X}$.mesh.var <- csc.jags\$BUGSoutput\$sims.list [['sd.mesh'] ] ${ }^{-2}$

X.protein.var <- csc.jags\$BUGSoutput\$sims.list [['sd.protein']] 2

X.chl.var <- csc.jags\$BUGSoutput\$sims.list [['sd.chl'] ] 2

X.temp.var <- csc.jags\$BUGSoutput\$sims.list [['sd.temp'] ] -2

X.cruise.var <- csc.jags\$BUGSoutput\$sims.list[['sd.cruise'] ]-2

X.int.var <- csc.jags\$BUGSoutput\$sims.list [['sd.int'] ] -2

R.var <- csc.jags\$BUGSoutput\$sims.list [['sd.res'] ] ${ }^{-2}$

R2.X<- (X.mesh.var + X.cruise.var + X.int.var + X.protein.var + X.chl.var + X.temp.var)/(X.mesh.var + X.cruise.var + X.int.var + X.protein.var + X.chl

$>$ R2.mesh<- ((X.mesh.var)/(X.mesh.var + X.cruise.var + X.int.var + X.protein.var + R.var))

R2.cruise<-((X.cruise.var)/(X.mesh.var + X.cruise.var + X.int.var + X.protein.var + X.chl.var + X.temp.var + R.var))

R2.int<-( X.int.var $) /(X . m e s h . v a r+X . c r u i s e . v a r+X . i n t . v a r+X . p r o t e i n . v a r+X . c h l . v a r+X . t e m p . v a r+R . v a r))$

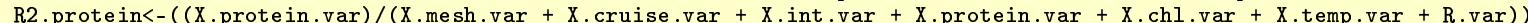

R2.chl<- ( X.chl.var $) /(X . m e s h . v a r+X . c r u i s e . v a r+X . i n t . v a r+X . c h l . v a r+X . c h l . v a r+X . t e m p . v a r+R . v a r))$

R2.temp<-((X.temp.var)/(X.mesh.var + X.cruise.var + X.int.var + X.temp.var + X.chl.var + X.temp.var + R.var ))

R2.resid <- ((R.var)/(X.mesh.var + X.cruise.var + X.int.var + X.protein.var + X.chl.var + X.temp.var + R.var))

a<-cbind(Residual=R.var, 'Temperature'=X.temp.var, 'Chlorophyll'=X.chl.var, 'Protein'=X.protein.var, 'Mesh $\mathrm{x}$ Cruise'=X.int.var, Cruise=X.cruise.var, sdtab <- cbind(Name=c ('Residuals', 'Temperature', 'Chlorophyll', 'Protein', 'Mesh x Cruise', 'Cruise', 'Mesh'), adply(a, 2, function(x) \{ data.frame $(\operatorname{Mean}=m e a n(x), \operatorname{Median}=\operatorname{median}(x)$, $\operatorname{HPDinterval}(\operatorname{as} \cdot \operatorname{mcmc}(\mathrm{x}))$, HPDinterval $(\operatorname{as} . \operatorname{mcmc}(\mathrm{x}), \mathrm{p}=0.68)$ )

\}))

a<-cbind(Residual=R2 .resid, 'Temperature'=R2.temp, 'Chlorophyll'=R2.chl, 'Protein'=R2.protein, 'Mesh x Cruise'=R2.int, Cruise=R2 .cruise, Mesh=R2.mesh R2tab <- cbind(Name=c('Residuals', 'Temperature', 'Chlorophyll', 'Protein', 'Mesh x Cruise', 'Cruise', 'Mesh'), adply(a, 2, function(x) \{ data.frame $(\operatorname{Mean}=\operatorname{mean}(\mathrm{x}), \operatorname{Median}=\operatorname{median}(\mathrm{x}), \operatorname{HPDinterval}(\operatorname{as} . \mathrm{mcmc}(\mathrm{x})), \operatorname{HPDinterval}(\operatorname{as} . \mathrm{mcmc}(\mathrm{x}), \mathrm{p}=0.68))$ \}))

sdtab\$R2 <- round $(\mathrm{R} 2$ tab\$Median, 3)

sdtab\$Name <- factor (sdtab\$Name, levels=c('Residuals', 'Temperature', 'Chlorophyll', 'Protein', 'Mesh x Cruise', 'Cruise', 'Mesh'))

sdtab <- subset $($ sdtab, select $=-\mathrm{X} 1)$

print(xtable(sdtab[7:1,], caption='Mean, median, (lower and upper) and highest probability density intervals of finite-population standard deviation \% latex table generated in R 3.1.0 by xtable 1.7-4 package \% Thu May 7 08:35:41 2015 
Table S2. Mean, median, (lower and upper) and highest probability density intervals of finite-population standard deviations as well as marginal $R^{2}$ approximations.

\begin{tabular}{lrrrrrrr}
\hline Name & Mean & Median & lower & upper & lower.1 & upper.1 & R2 \\
\hline Mesh & 696.72 & 562.60 & 0.25 & 1816.46 & 1.04 & 838.47 & 0.12 \\
Cruise & 1362.91 & 1276.16 & 211.12 & 2682.70 & 541.59 & 1795.14 & 0.25 \\
Mesh x Cruise & 1053.77 & 955.49 & 219.58 & 2120.44 & 389.81 & 1332.99 & 0.19 \\
Protein & 90.49 & 50.39 & 0.00 & 299.07 & 0.00 & 100.07 & 0.01 \\
Chlorophyll & 48.05 & 21.42 & 0.00 & 195.23 & 0.00 & 44.91 & 0.00 \\
Temperature & 45.01 & 21.43 & 0.00 & 169.90 & 0.00 & 45.69 & 0.00 \\
Residuals & 2085.21 & 2067.31 & 1891.24 & 2328.62 & 1938.82 & 2154.55 & 0.40 \\
\hline
\end{tabular}

p1 <- $\operatorname{ggplot}(\mathrm{sdtab}$, aes $(\mathrm{y}=$ Name, $\mathrm{x}=$ Median $))+$

geom_vline ( $x$ intercept $=0$, linetype='dashed' $)+$

geom_errorbarh (aes (xmin=lower, xmax=upper), height=0)+

geom_errorbarh (aes $(x \min =1$ lower.1, xmax=upper .1 ), height=0, size=2)+

geom_point (size $=3$, shape $=21$, fill='white') +

geom_text (aes (label=R2, y=as.numeric (Name)+0.3), position=position_dodge (height=1), size=3)+

scale_x_continuous ('Finite-population standard deviation') +

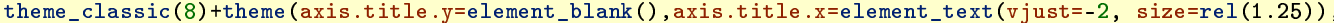

$\operatorname{print}(\mathrm{p} 1)$ plot.margin=unit $(c(0,0,2,2)$, 'lines'))

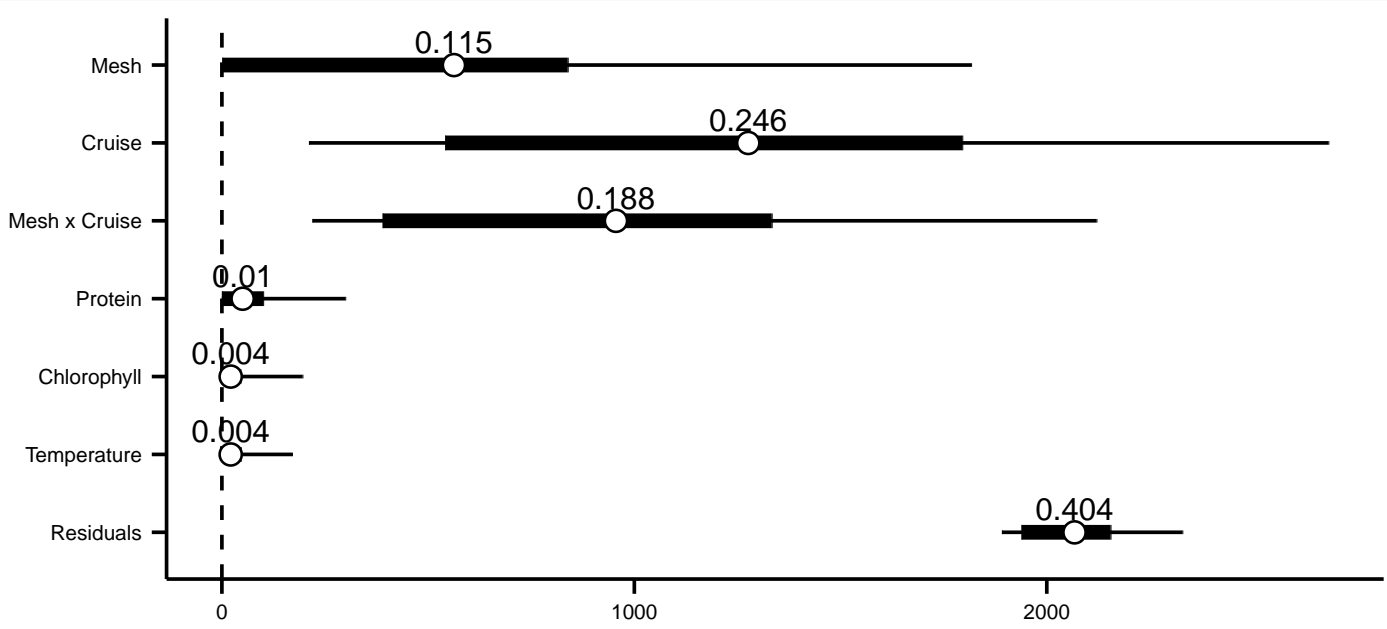

Finite-population standard deviation

Figure P. Median (lower and upper) highest probability density intervals of finite-population standard deviations. $R^{2}$ approximations above medians. 
newdata <- expand.grid(Mesh=levels (csc\$Mesh), Cruise=levels (csc\$Cruise))

$>$ newdata $\$$ Protein <- mean (csc\$Protein, na.rm=TRUE)

$>$ newdata $\$ C h l<-\operatorname{mean}(\csc \$ C h l$, na.rm=TRUE)

$>$ newdata\$Temp <- mean (csc\$Temp, na.rm=TRUE)

$>$ Xmat <- model.matrix ( Mesh*Cruise, data=newdata)

$>$ XXmat <- model.matrix ( $\log ($ Protein $)+\log (\mathrm{Ch} 1)+$ Temp, data=newdata) $[,-1]$

$>$ coef <- csc.jags\$BUGSoutput\$sims.list [ ['beta']]

fit <- coef $\% * \%$ t(Xmat) + csc.jags $\$$ BUGSoutput $\$$ sims.list [['gamma'] ] \%*\% t (XXmat)

$>$ library (plyr)

$>$ newdata $<$ - cbind(newdata, adply(fit, 2, function(x) \{

data.frame $(\operatorname{Mean}=\operatorname{mean}(x), \operatorname{Median}=\operatorname{median}(x), \operatorname{HPDinterval}(\operatorname{as} . \operatorname{mcmc}(x)), \operatorname{HPDinterval}(\operatorname{as} . m c m c(x), p=0.68))$ \}))

$>$ \#newdata

\#Comparisons

MCMCsum <- function(x) \{

data. frame (Median=median ( $x$, na.rm=TRUE),

t (quantile ( $x$, na.rm=TRUE))

HPDinterval (as . mcmc (x))

$+\}$ HPDinterval (as . $\operatorname{mcmc}(x), p=0.68)$ )

library (multcomp)

$>$ ndata $<-$ newdata

$>$ ndata $<-$ subset (ndata, Cruise=='1')

Xmat <- contrMat (n=rep(1,nrow(ndata)), type="Tukey") \%*\% model.matrix ( Mesh*Cruise, ndata, xlev=list (Mesh=levels (csc $\$$ Mesh), Cruise=levels (csc $\$$ Cruise)

$>$ wch <- ldply(strsplit (rownames (Xmat)," - "), as.numeric)

$>$ rownames (Xmat) <- paste (interaction(newdata\$Mesh, newdata $\$ C r u i s e)[w c h[, 1]], "-"$,interaction (newdata $\$$ Mesh, newdata $\$ C r u i s e)[w c h[, 2]])$

$>$ pairwise.comps <- coef $\% * \%$ t (Xmat)

$>$ comp.mesh_Cruise1<-adply (pairwise.comps, 2 , MCMCsum)

$>$ ndata $<-$ newdata

$>$ ndata $<-$ subset (ndata, Cruise=='2')

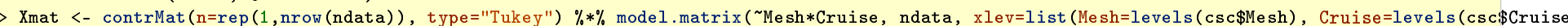

$>$ wch <- ldply(strsplit (rownames (Xmat)," - "), as .numeric)

$>$ rownames (Xmat) <- paste (interaction(newdata $\$$ Mesh, newdata $\$$ Cruise) [wch [,1]], "-", interaction (newdata $\$$ Mesh, newdata $\$ C r u i s e)[w c h[, 2]]$ )

$>$ pairwise.comps <- coef $\% * \%$ t (Xmat)

$>$ comp.mesh_Cruise2<-adply (pairwise.comps,2, MCMCsum)

ndata $<-$ newdata

ndata $<-$ subset (ndata, Cruise==' $\left.3^{\prime}\right)$

Xmat <- contrMat (n=rep (1,nrow (ndata)), type="Tukey") \%*\% model.matrix( ${ }^{M e s h} *$ Cruise, ndata, xlev=list (Mesh=levels (csc $\$$ Mesh), Cruise=levels (csc $\left.\$ C r u i s e\right)$

$>$ wch <- ldply(strsplit (rownames (Xmat)," - "), as.numeric)

$>\operatorname{rownames}($ Xmat) <- paste (interaction(newdata $\$$ Mesh, newdata $\$ C r u i s e)[w c h[, 1]], "-"$, interaction (newdata $\$$ Mesh, newdata $\$ C r u i s e)[w c h[, 2]]$ )

pairwise.comps <- coef $\% * \%$ t (Xmat)

comp.mesh_Cruise3<-adply (pairwise.comps, 2 , MCMCsum)

ndata $<-$ newdata

ndata $<-$ subset (ndata, Cruise==' 4')

Xmat <- $\operatorname{contrMat(n=rep(1,nrow(ndata)),~type="Tukey")~\% *\% ~model.matrix~(~Mesh*Cruise,~ndata,~xlev=list~(Mesh=levels~(csc~} \$$ Mesh), Cruise=levels (csc $\$ C r u i s e)$

$>$ wch <- ldply(strsplit (rownames(Xmat)," - "), as.numeric)

rownames (Xmat) <- paste(interaction(newdata\$Mesh, newdata $\$ C r u i s e)[w c h[, 1]], "-"$, interaction (newdata $\$$ Mesh, newdata $\$ C r u i s e)[w c h[, 2]]$ )

pairwise.comps <- coef $\% * \%$ t (Xmat)

comp.mesh_Cruise4<-adply (pairwise.comps, 2, MCMCsum)

comp.mesh <- rbind(data.frame (Cruise=1, comp.mesh_Cruise1) data frame (Cruise $=2$, comp.mesh Cruise2) data.frame (Cruise=3, comp. mesh_Cruise 3 ) data.frame (Cruise $=4$, comp.mesh_Cruise4))

p1 <- ggplot (comp.mesh, aes ( $\mathrm{x}=$ Cruise, $\mathrm{y}=$ Median, color $=\mathrm{X} 1))+$ geom_hline (yintercept $=0$, linetype $=$ 'dashed') + geom_errorbar (aes (ymin=lower, ymax=upper), width=0, position=position_dodge (width=0.5)) + geom_errorbar (aes (ymin=lower.1, ymax=upper.1), width=0, size=2, position=position_dodge $($ width=0.5) $)+$ geom_point (size=3, shape=21, fill='white',position=position_dodge $($ width=0.5) $)$ +

scale_y_continuous('Median effect size') +

\#scale $x$ discrete ('Cruise number') t

scale_color_manual ('Mesh contrasts', breaks=c('150.1 - 73.1', '350.1 - 73.1','350.1 - 150.1'), labels=c('150 vs 73', coord_flip ()+theme_classic ()$+$

theme (axis.title.y=element_text ( $\operatorname{size}=r e l(1.25)$, vjust=2),

axis.title. $x=e l e m e n t$ text ( $\operatorname{size}=r e l(1.25)$, vjust $=-1)$,

plot . margin=unit $(c(0,0,1,1)$, 'lines' $))$

ndata <- newdata

ndata $<-$ subset (ndata, Mesh==' $73^{\prime}$ )

Xmat <- contrMat (n=rep (1, nrow (ndata)), type="Tukey") \%*\% model.matrix ( Mesh*Cruise, ndata, xlev=list (Mesh=levels (csc $\$$ Mesh), Cruise=levels (csc $\$$ Cruise) pairwise.comps <- coef $\% * \%$ t (Xmat)

comp.cruise_Mesh73<-adply (pairwise.comps,2, MCMCsum)

ndata $<-$ newdata

ndata<- $\operatorname{subset}\left(\right.$ ndata, Mesh==' $\left.150^{\prime}\right)$

Xmat <- contrMat (n=rep (1,nrow (ndata)), type="Tukey") \%*\% model.matrix( Mesh*Cruise, ndata, xlev=list (Mesh=levels (csc $\$$ Mesh), Cruise=levels (csc $\$ C r u i s e)$

pairwise.comps <- coef $\% * \%$ t (Xmat)

comp.cruise_Mesh150<-adply (pairwise.comps,2, MCMCsum)

ndata <- newdata

ndata<- subset (ndata, Mesh=='350')

Xmat <- contrMat (n=rep (1, nrow (ndata)), type="Tukey") \%*\% model.matrix ( Mesh*Cruise, ndata, xlev=list (Mesh=levels (csc $\$$ Mesh), Cruise=levels (csc $\$$ Cruise) pairwise.comps <- coef $\% * \%$ t (Xmat)

comp.cruise_Mesh350<-adply (pairwise.comps, 2, MCMCsum)

$>$ comp.cruise <- rbind(data.frame (Mesh=73, comp.cruise_Mesh73)

data.frame (Mesh=150, comp. cruise_Mesh150) data.frame (Mesh=350, comp. cruise_Mesh350)) 


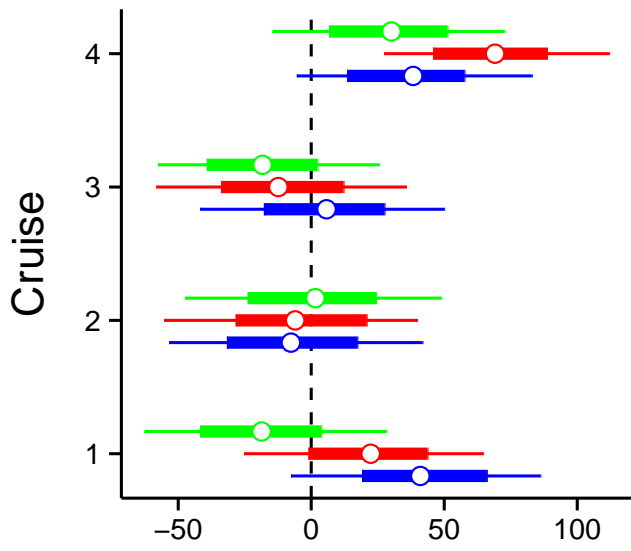

Median effect size

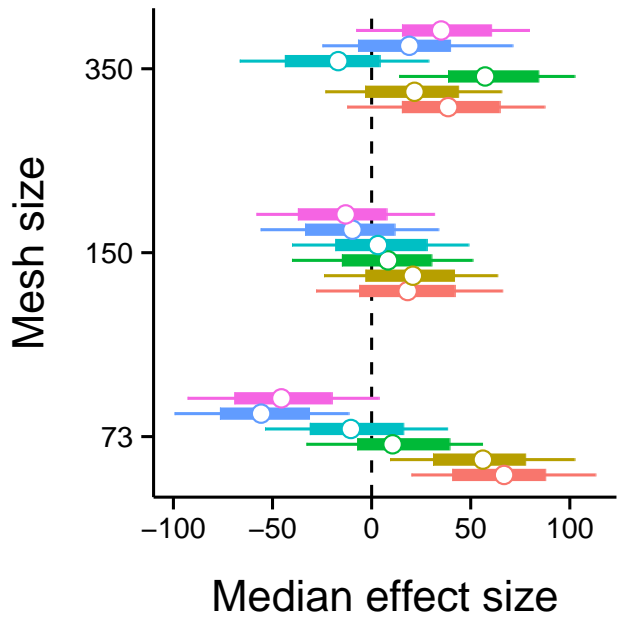

Cruise contrasts

IOI 2 - 1

IO= $3-1$

- 4 - 1

10. $3-2$

IO- $4-2$

IO- $4-3$

Figure Q. plot of chunk AARScontrasts 


\subsection{Kimberley}

\subsubsection{ETS}

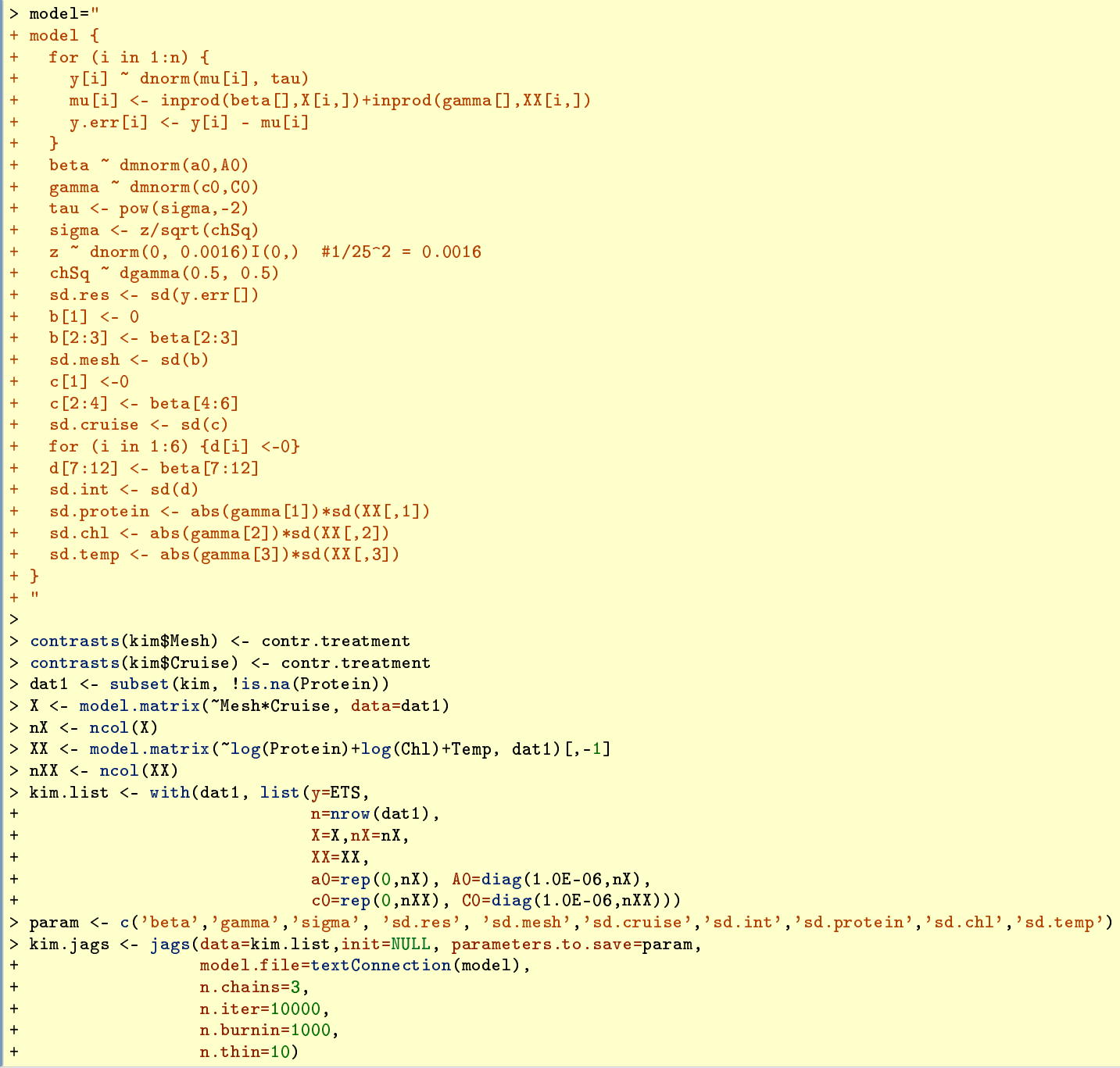

Compiling model graph

Resolving undeclared variables

Allocating nodes

Graph Size: 6558

Initializing model

kim.jags

Inference for Bugs model at "5", fit using jags,

3 chains, each with 10000 iterations (first 1000 discarded), n.thin $=10$

n.sims $=2700$ iterations saved

mu.vect sd.vect $\quad 2.5 \% \quad 25 \% \quad 50 \% \quad 75 \% \quad 97.5 \%$ Rhat n.eff

beta[1] $\quad-408.238 \quad 663.701 \quad-1705.464 \quad-859.748 \quad-422.093 \quad 37.516 \quad 890.094 \quad 1.001 \quad 2700$

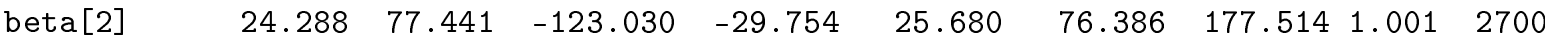

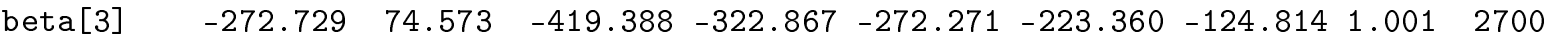

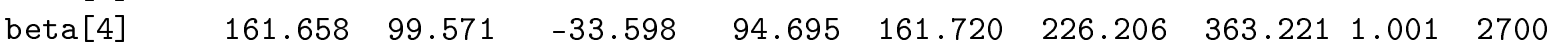

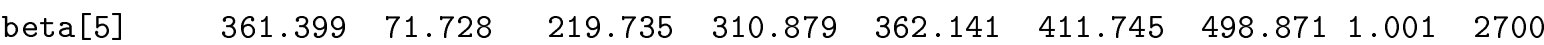




\begin{tabular}{|c|c|c|c|c|c|c|c|c|c|}
\hline eta [6] & 238.714 & 91.122 & 65.208 & 177.459 & 235.831 & 300.628 & 420.637 & 1.001 & 2700 \\
\hline seta $[7]$ & 36.768 & 91.606 & -145.106 & -24.503 & 35.148 & 98.409 & 215.701 & 1.001 & 2500 \\
\hline eta [8] & -243.878 & 99.338 & -445.940 & -308.708 & -244.888 & -177.413 & -47.409 & 1.001 & 2700 \\
\hline eta [9] & -39.248 & 99.268 & -240.870 & -104.390 & -39.543 & 26.599 & 159.832 & 1.001 & 2700 \\
\hline $\mathrm{ta}[10]$ & -285.742 & 97.382 & -469.397 & -352.215 & -285.802 & -220.899 & -93.263 & 1.001 & 2700 \\
\hline & -308.403 & 96.098 & -485.747 & -375.097 & -311.573 & -243.547 & -110.836 & 1.000 & 2700 \\
\hline eta [12] & -239.085 & 110.587 & -456.289 & -313.517 & -241.219 & -164.143 & -27.326 & 1.000 & 2700 \\
\hline & -121.640 & 111.502 & -348.982 & -194.514 & -122.490 & -46.978 & 91.013 & 1.001 & 2700 \\
\hline eta $[14]$ & -155.749 & 103.927 & -355.795 & -224.795 & -156.364 & -82.851 & 44.279 & 1.001 & 2200 \\
\hline & -25.127 & 102.848 & -231.842 & -93.226 & -24.245 & 44.323 & 172.111 & 1.001 & 2700 \\
\hline $\mathrm{amn}$ & 21.246 & 17.153 & -11.727 & 9.339 & 21.471 & 32.696 & 100 & 1.003 & \\
\hline $\mathrm{amm}$ & 22.561 & 42.469 & -59.310 & -6.311 & 21.976 & 50.606 & 108.593 & 1.001 & 2700 \\
\hline & 34.099 & 22.356 & -10.261 & 18.968 & & 49.279 & 77.478 & & \\
\hline 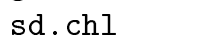 & 23.201 & 17.302 & 1.056 & 9.524 & 19.796 & 33.431 & 65.477 & 1.001 & 2700 \\
\hline ALN & 161.458 & 30.193 & 101.846 & 141.218 & 161.270 & 181.931 & 221.645 & 1.001 & 2700 \\
\hline 140 & 145.934 & 36.141 & 80.869 & 120.319 & 144.228 & 169.427 & 219.618 & 1.001 & 2700 \\
\hline mesh & 169.497 & 35.150 & 101.661 & 146.409 & 168.542 & 193.135 & 238.492 & 1.001 & 2700 \\
\hline d.protein & 25.256 & 16.174 & 1. 216 & 12.117 & 23.807 & 36.223 & 59.422 & 1.001 & 2100 \\
\hline & 205.882 & 2.758 & 201.448 & 203.878 & 205.606 & 207.521 & 212.032 & 1.001 & 2700 \\
\hline & 54.424 & 31.391 & 3.012 & 30.039 & 53.109 & 75.933 & 119.384 & 1.005 & 1900 \\
\hline & 206.026 & 9.408 & 188.862 & 199.482 & 205.422 & 212.108 & 226.045 & 1.002 & 1200 \\
\hline evia & 3441.492 & 6.381 & 3431.047 & 3437.029 & 3440.715 & 3445.050 & 3456.099 & 1.001 & 2700 \\
\hline
\end{tabular}

For each parameter, $n$.eff is a crude measure of effective sample size, and Rhat is the potential scale reduction factor (at convergence, Rhat=1).

DIC info (using the rule, $\mathrm{pD}=\operatorname{var}($ deviance) $/ 2$ )

$\mathrm{pD}=20.4$ and $\mathrm{DIC}=3461.9$

DIC is an estimate of expected predictive error (lower deviance is better).

\#\# Finite-population standard deviations

X.mesh.var <- kim.jags\$BUGSoutput\$sims.list [['sd.mesh']]-2

X.protein.var <- kim.jags\$BUGSoutput\$sims.list [['sd.protein']]-2

X.chl.var <- kim.jags\$BUGSoutput\$sims.list [['sd.chl'] ] ] -2

X.temp.var <- kim.jags\$BUGSoutput\$sims.list [['sd.temp'] ] -2

X.cruise.var <- kim.jags\$BUGSoutput\$sims.list[['sd.cruise']] 2

X.int.var <- kim.jags\$BUGSoutput\$sims.list[['sd.int'] ] -2

R.var <- kim.jags\$BUGSoutput\$sims.list [['sd.res'] ] -2

R2.X<- (X.mesh.var + X.cruise.var + X.int.var + X.protein.var + X.chl.var + X.temp.var)/(X.mesh.var + X.cruise.var + X.int.var + X.protein.var + X.ch

R2.mesh<-((X.mesh.var)/(X.mesh.var + X.cruise.var + X.int.var + X.protein.var + R.var))

R2.cruise<-((X.cruise.var)/(X.mesh.var + X.cruise.var + X.int.var + X.protein.var + X.chl.var + X.temp.var + R.var ))

R2.int<- ( X.int.var $) /(X$.mesh.var + X.cruise.var + X.int.var + X.protein.var + X.chl.var + X.temp.var + R.var ) )

R2.protein<- ( X.protein.var $) /(X . m e s h . v a r+X . c r u i s e . v a r+X . i n t . v a r+X . p r o t e i n . v a r+X . c h l . v a r+X . t e m p . v a r+R . v a r))$

R2.chl<- ( X.chl.var)/(X.mesh.var + X.cruise.var + X.int.var + X.chl.var + X.chl.var + X.temp.var + R.var $))$

R2.temp<-((X.temp.var)/(X.mesh.var + X.cruise.var + X.int.var + X.temp.var + X.chl.var + X.temp.var + R.var))

R2.resid <- ((R.var)/(X.mesh.var + X.cruise.var + X.int.var + X.protein.var + X.chl.var + X.temp.var + R.var))

a<-cbind(Residual=R.var, 'Temperature'=X.temp.var, 'Chlorophyll'=X.chl.var, 'Protein'=X.protein.var, 'Mesh $\mathrm{x}$ Cruise'=X.int.var, Cruise=X.cruise.var, sdtab <- cbind(Name=c('Residuals', 'Temperature', 'Chlorophyll', 'Protein', 'Mesh x Cruise', 'Cruise', 'Mesh'), adply(a, 2, function(x) \{ data.frame $(\operatorname{Mean}=\operatorname{mean}(\mathrm{x}), \operatorname{Median}=\operatorname{median}(\mathrm{x})$, $\operatorname{HPDinterval}(\operatorname{as} \cdot \operatorname{mcmc}(\mathrm{x}))$, $\operatorname{HPDinterval}(\operatorname{as} . \operatorname{mcmc}(\mathrm{x}), \mathrm{p}=0.68)$ ) \}))

a<-cbind(Residual=R2.resid, 'Temperature'=R2.temp, 'Chlorophyll'=R2.chl, 'Protein'=R2.protein, 'Mesh $x$ Cruise'=R2.int, Cruise=R2 .cruise, Mesh=R2 .mes R2tab <- cbind(Name=c('Residuals', 'Temperature', 'Chlorophyll', 'Protein', 'Mesh x Cruise', 'Cruise', 'Mesh'), adply(a, 2, function(x) \{ data.frame $($ Mean $=\operatorname{mean}(\mathrm{x}), \operatorname{Median}=\operatorname{median}(\mathrm{x})$, $\operatorname{HPDinterval}(\operatorname{as} \cdot \operatorname{mcmc}(\mathrm{x}))$, $\operatorname{HPDinterval}(\operatorname{as} . \mathrm{mcmc}(\mathrm{x}), \mathrm{p}=0.68))$ \}))

sdtab\$R2 <- round(R2tab\$Median,3)

sdtab\$Name <- factor (sdtab\$Name, levels=c('Residuals', 'Temperature', 'Chlorophyll', 'Protein', 'Mesh x Cruise', 'Cruise', 'Mesh'))

sdtab $<-$ subset $($ sdtab, select $=-\mathrm{X} 1)$

print(xtable(sdtab[7:1,], caption='Mean, median, (lower and upper) and highest probability density intervals of finite-population standard deviation \% latex table generated in R 3.1.0 by xtable 1.7-4 package \% Thu May 7 08:35:43 2015 
Table S3. Mean, median, (lower and upper) and highest probability density intervals of finite-population standard deviations as well as marginal $R^{2}$ approximations.

\begin{tabular}{lrrrrrrr}
\hline Name & Mean & Median & lower & upper & lower.1 & upper.1 & R2 \\
\hline Mesh & 29964.17 & 28406.29 & 7778.75 & 52971.70 & 16229.73 & 39320.80 & 0.24 \\
Cruise & 26980.04 & 26007.99 & 9420.74 & 46329.49 & 16700.66 & 35664.58 & 0.21 \\
Mesh x Cruise & 22602.37 & 20801.81 & 5278.46 & 44404.80 & 9133.18 & 28755.93 & 0.17 \\
Protein & 899.37 & 566.79 & 0.00 & 2928.71 & 0.00 & 1041.05 & 0.00 \\
Chlorophyll & 837.54 & 391.90 & 0.00 & 3254.10 & 0.00 & 824.17 & 0.00 \\
Temperature & 3946.92 & 2820.52 & 0.00 & 11661.24 & 0.00 & 4722.05 & 0.02 \\
Residuals & 42394.82 & 42273.88 & 40408.95 & 44654.92 & 40887.71 & 43050.46 & 0.34 \\
\hline
\end{tabular}

p1 <- $\operatorname{ggplot}(\mathrm{sdtab}$, aes $(\mathrm{y}=$ Name, $\mathrm{x}=$ Median $))+$

geom_vline ( $x$ intercept $=0$, linetype='dashed' $)+$

geom_errorbarh (aes (xmin=lower, xmax=upper), height $=0)+$

geom_errorbarh(aes (xmin=lower.1, xmax=upper.1), height=0, size=2)+

geom_point ( $\operatorname{size}=3$, shape $=21$, fill='white') +

geom_text (aes (label=R2, $y=$ as .numeric (Name) +0.3), position=position_dodge (height=1), size $=3$ ) +

scale_x_continuous ('Finite-population standard deviation') +

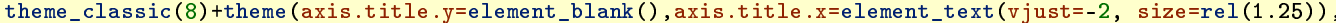
plot.margin=unit $(c(0,0,2,2)$, 'lines')

print (p1)

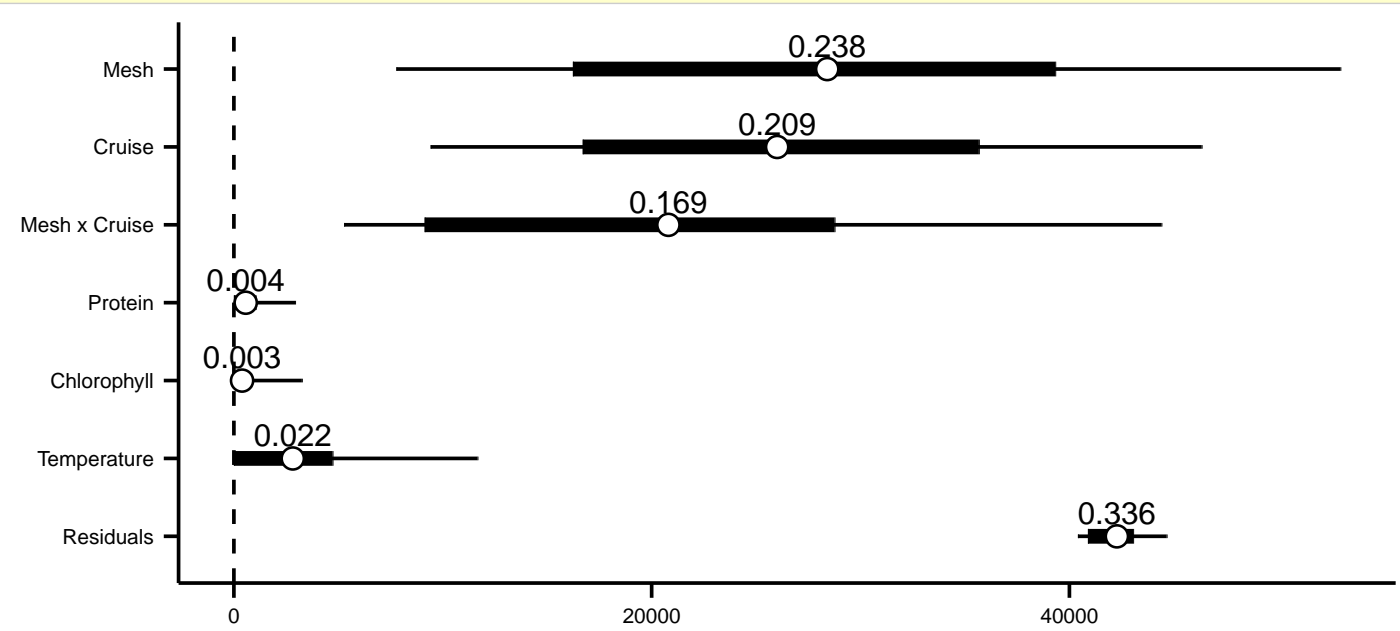

Finite-population standard deviation

Figure R. Median (lower and upper) highest probability density intervals of finite-population standard deviations. $R^{2}$ approximations above medians. 
newdata <- expand.grid(Mesh=levels(kim\$esh), Cruise=levels (kim\$Cruise))

$>$ newdata $\$$ Protein <- mean (kim\$Protein, na.rm=TRUE)

$>$ newdata $\$ C h l<-$ mean(kim $\$ C h l$, na.rm=TRUE)

$>$ newdata $\$$ Temp <- mean (kim\$Temp, na.rm=TRUE)

$>$ Xmat <- model.matrix ( ${ }^{M e s h} *$ Cruise, data=newdata)

$>$ XXmat <- model.matrix ( $\log ($ Protein $)+\log (\mathrm{Ch} l)+$ Temp, data=newdata) $[,-1]$

$>$ coef <- kim.jags\$BUGSoutput\$sims.list [['beta']]

fit <- coef $\% \%$ t(Xmat) + kim.jags $\$ B U G S o u t p u t \$ s i m s . l i s t[$ ['gamma']] \%*\% t (XXmat)

$>$ library (plyr)

$>$ newdata $<$ - cbind(newdata, adply(fit, 2, function(x) \{

data.frame $(\operatorname{Mean}=\operatorname{mean}(x), \operatorname{Median}=\operatorname{median}(x), \operatorname{HPDinterval}(\operatorname{as} . \operatorname{mcmc}(x)), \operatorname{HPDinterval}(\operatorname{as} . m c m c(x), p=0.68))$ \}))

$>$ \#newdata

\#Comparisons

MCMCsum <- function( $\mathrm{x})$ \{

data.frame (Median=median ( $x$, na.rm=TRUE),

t (quantile ( $x, n a \cdot r m=T R U E)$ )

HPDinterval (as . mcmc ( $x$ )) ,

\} HPDinterval (as.mcmc $(x), p=0.68)$ )

library (multcomp)

ndata $<-$ newdata

ndata $<-$ subset (ndata, Cruise=='1')

$>$ Xmat <- contrMat (n=rep (1,nrow(ndata)), type="Tukey") \%*\% model.matrix( Mesh*Cruise, ndata, xlev=list (Mesh=levels(kim $\$$ Mesh), Cruise=levels (kim

$>$ wch <- ldply(strsplit (rownames (Xmat)," - "), as.numeric)

$>\operatorname{rownames}($ Xmat) <- paste (interaction(newdata\$Mesh, newdata $\$ C r u i s e)[w c h[, 1]], "-"$, interaction (newdata $\$$ Mesh, newdata $\$ C r u i s e)[w c h[, 2]])$

pairwise.comps <- coef $\% * \%$ t (Xmat)

$>$ comp.mesh_Cruise1<-adply (pairwise.comps, 2 , MCMCsum)

$>$ ndata $<-$ newdata

ndata<- subset (ndata, Cruise=='2')

Xmat <- contrMat (n=rep(1,nrow (ndata)), type="Tukey") \%*\% model.matrix( Mesh*Cruise, ndata, xlev=list (Mesh=levels(kim\$Mesh), Cruise=levels (kimpCruise)

$>$ wch <- ldply(strsplit (rownames (Xmat)," - "), as .numeric)

$>$ rownames (Xmat) <- paste (interaction(newdata $\$$ Mesh, newdata $\$$ Cruise) [wch [,1]], "-", interaction (newdata $\$$ Mesh, newdata $\$ C r u i s e)[w c h[, 2]]$ )

$>$ pairwise.comps <- coef $\% * \%$ t (Xmat)

comp.mesh_Cruise2<-adply (pairwise.comps,2, MCMCsum)

ndata $<-$ newdata

ndata $<-$ subset (ndata, Cruise==' $\left.3^{\prime}\right)$

Xmat <- contrMat (n=rep (1,nrow (ndata)), type="Tukey") \%*\% model.matrix( ${ }^{M e s h * C r u i s e, ~ n d a t a, ~ x l e v=l i s t(M e s h=l e v e l s(k i m ~} \$$ Mesh), Cruise=levels (kim $\$$ Cruise)

$>$ wch <- ldply(strsplit (rownames(Xmat)," - "), as.numeric)

$>\operatorname{rownames}($ Xmat) <- paste (interaction(newdata\$Mesh, newdata $\$ C r u i s e)[w c h[, 1]], "-"$, interaction (newdata $\$$ Mesh, newdata $\$ C r u i s e)[w c h[, 2]])$

pairwise.comps <- coef $\% * \%$ t (Xmat)

comp.mesh_Cruise3<-adply (pairwise.comps, 2, MCMCsum)

ndata <- newdata

ndata $<-$ subset (ndata, Cruise=='4')

Xmat <- contrMat (n=rep(1,nrow(ndata)), type="Tukey") \%*\% model.matrix( Mesh*Cruise, ndata, xlev=list (Mesh=levels(kim $\$$ Mesh), Cruise=levels (kim $\$$ Cruise)

$>$ wch <- ldply(strsplit (rownames(Xmat)," - "), as.numeric)

rownames (Xmat) <- paste(interaction(newdata $\$$ Mesh, newdata $\$ C r u i s e)[w c h[, 1]], "-"$, interaction (newdata $\$$ Mesh, newdata $\$ C r u i s e)[w c h[, 2]]$ )

pairwise.comps <- coef $\% * \%$ t (Xmat)

$>$ comp.mesh_Cruise4<-adply (pairwise.comps, 2 , MCMCsum)

$>$ ndata $<-$ newdata

ndata $<-$ subset (ndata, Cruise $==' 5$ ')

Xmat <- contrMat (n=rep (1,nrow(ndata)), type="Tukey") \%*\% model.matrix( Mesh*Cruise, ndata, xlev=list (Mesh=levels(kim $\$$ Mesh), Cruise=levels (kim $\$$ Cruise)

$>$ wch <- ldply(strsplit (rownames(Xmat)," - "), as .numeric)

$>\operatorname{rownames}($ Xmat) <- paste (interaction(newdata $\$$ Mesh, newdata $\$ C r u i s e)[w c h[, 1]], "-"$, interaction (newdata $\$ M e s h$, newdata $\$ C r u i s e)[w c h[, 2]])$

pairwise.comps <- coef $\% * \%$ t (Xmat)

comp.mesh_Cruise5<-adply (pairwise.comps,2, MCMCsum)

comp.mesh <- rbind(data.frame(Cruise=1, comp.mesh_Cruise1) data. frame (Cruise $=2$, comp. mesh_Cruise 2) data. frame (Cruise $=3$, comp. mesh_Cruise 3 ) data.frame (Cruise $=4$, comp.mesh_Cruise 4 ), data.frame (Cruise =5, comp.mesh_Cruise5))

$\mathrm{p} 1<-\operatorname{ggplot}(\operatorname{comp} . \mathrm{mesh}$, aes $(\mathrm{x}=$ Cruise, $\mathrm{y}=$ Median, color $=\mathrm{X} 1))+$ geom_hline (yintercept $=0$, linetype $=$ 'dashed') +

geom_errorbar(aes (ymin=lower, ymax=upper), width=0, position=position_dodge (width=0.5)) +

geom_errorbar (aes (ymin=lower.1, ymax=upper.1), width=0, size=2, position=position_dodge $($ width=0.5)) + geom_point ( $\operatorname{size}=3$, shape $=21$, fill=' white', position=position_dodge $($ width $=0.5))+$ scale_y_continuous('Median effect size') +

\#scale $x$ discrete ('Cruise number') +

scale_color_manual ('Mesh contrasts', breaks=c('150.1 - 73.1', '350.1 - 73.1', '350.1 - 150.1'), labels=c('150 vs 73', coord_flip()+theme_classic()+

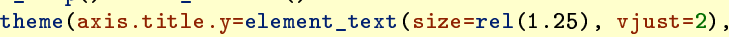
axis.title. $x=e l e m e n t$ text $(\operatorname{size}=r e l(1.25)$, vjust $=-1)$ plot.margin=unit $(c(0,0,1,1)$, 'lines' $))$

ndata $<-$ newdata

ndata<- subset (ndata, Mesh=='73')

Xmat <- $\operatorname{contrMat}(\mathrm{n}=\operatorname{rep}(1$, nrow (ndata)), type="Tukey") \%*\% model.matrix ( Mesh*Cruise, ndata, xlev=list (Mesh=levels (kim $\$$ Mesh), Cruise=levels (kim 1 Cruise pairwise.comps <- coef $\% * \%$ t (Xmat)

comp.cruise_Mesh73<-adply (pairwise.comps, 2 , MCMCsum)

ndata $<-$ newdata

ndata $<-\operatorname{subset}($ ndata, Mesh=='150')

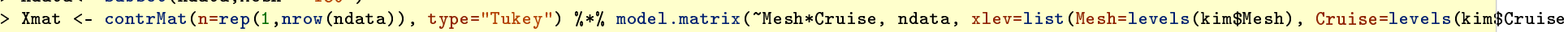
pairwise.comps <- coef $\% * \%$ t (Xmat)

comp.cruise_Mesh150<-adply (pairwise.comps, 2, MCMCsum) 

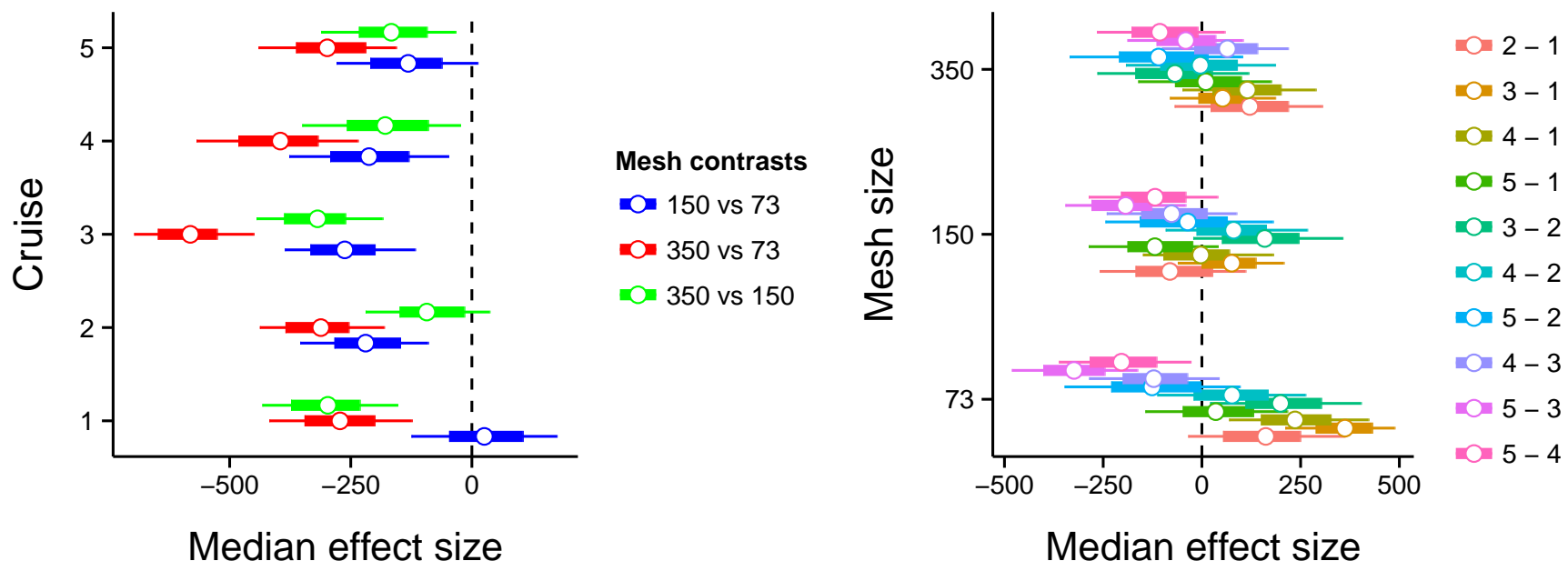

Figure S. plot of chunk ETSKimcontrasts 


\subsubsection{AARS}

$>\operatorname{model}={ }^{\prime}$

model \{

for ( $i$ in $1: n)\{$

$y[i] \sim \operatorname{dnorm}(\mathrm{mu}[i], \mathrm{tau})$

$\mathrm{mu}[\mathrm{i}]<-\operatorname{inprod}(\operatorname{beta}[], \mathrm{X}[\mathrm{i}])+,\mathrm{inprod}(\operatorname{gamma}[], \mathrm{XX}[\mathrm{i}]$,

\}

$y \cdot \operatorname{err}[i]<-y[i]-\operatorname{mu}[i]$

beta dmnorm $(\mathrm{a} 0, \mathrm{AO})$

gamma dmnorm $(\mathrm{cO}, \mathrm{CO})$

tau <- pow (sigma, -2)

sigma $<-\mathrm{z} / \mathrm{sqrt}(\mathrm{chSq})$

$z \sim \operatorname{dnorm}(0,0.0016) \mathrm{I}(0) \quad \# ,1 / 25^{-2}=0.0016$

$\mathrm{chSq} \sim \operatorname{dgamma}(0.5,0.5)$

sd.res <- $\operatorname{sd}(\mathrm{y} \cdot \operatorname{err}[])$

$\mathrm{b}[1]<-0$

$\mathrm{b}[2: 3]<-\operatorname{beta}[2: 3]$

sd.mesh $<-$ sd $(b)$

$c[1]<-0$

$c[2: 4]<-\operatorname{beta}[4: 6]$

sd.cruise <- sd (c)

for ( $i$ in $1: 6) \quad$ d [i] $<-0\}$

$d[7: 12]<-$ beta $[7: 12]$

sd.int $<-\operatorname{sd}(d)$

sd.protein <- $\operatorname{abs}(\operatorname{gamma}[1]) * \operatorname{sd}(\mathrm{XX}[, 1])$

sd.chl $<-$ abs $($ gamma $[2]) * \operatorname{sd}(\mathrm{XX}[, 2])$

\}

sd.temp <- abs (gamma [3])*sd $(X X[, 3])$

$>$ contrasts $(\mathrm{kim} \$ \mathrm{Mesh})<-$ contr.treatment

$>$ contrasts (kim\$Cruise) <- contr.treatment

dat1 <- subset(kim, ! is.na(Protein))

$>\mathrm{X}<-$ model.matrix ( Mesh $*$ Cruise, data=dat1)

$>\mathrm{nX}<-\mathrm{ncol}(\mathrm{X})$

$>\mathrm{XX}<-$ model.matrix $(\sim \log (\operatorname{Prote} i n)+\log (\mathrm{Ch} 1)+\operatorname{Temp}, \operatorname{dat} 1)[,-1]$

$\mathrm{nXX}<-\mathrm{ncol}(\mathrm{XX})$

kim.list <- with(dat1, list (y=AARS

$\mathrm{n}=\mathrm{nrow}(\operatorname{dat} 1)$,
$\mathrm{X}=\mathrm{X}, \mathrm{nX}=\mathrm{nX}$,

$\mathrm{XX}=\mathrm{XX}$,

$\mathrm{a} 0=\operatorname{rep}(0, \mathrm{nX}), \mathrm{A} 0=\operatorname{diag}(1.0 \mathrm{E}-06, \mathrm{nX})$,

$\mathrm{c} 0=\operatorname{rep}(0, \mathrm{nXX}), \quad \mathrm{C} 0=\operatorname{diag}(1.0 \mathrm{E}-06, \mathrm{nXX})))$

$>$ param <- c('beta', 'gamma', 'sigma', 'sd.res', 'sd.mesh', 'sd.cruise', 'sd.int', 'sd.protein', 'sd.chl', 'sd.temp')

$>$ kim.jags <- jags (data=kim.list,init=NULL, parameters.to.save=param,

$$
\begin{aligned}
& \text { model } . \text { file }=\text { tex } \\
& \text { n. chains }=3 \text {, } \\
& \text { n.iter }=10000 \text {, } \\
& \text { n. burnin }=1000 \text {, }
\end{aligned}
$$

n.thin=10)

Compiling model graph

Resolving undeclared variables

Allocating nodes

Graph Size: 6558

Initializing model

kim.jags

Inference for Bugs model at "5", fit using jags,

3 chains, each with 10000 iterations (first 1000 discarded), n.thin $=10$

n.sims $=2700$ iterations saved

mu.vect sd.vect $\quad 2.5 \% \quad 25 \% \quad 50 \% \quad 75 \% \quad 97.5 \% \quad$ Rhat n.eff

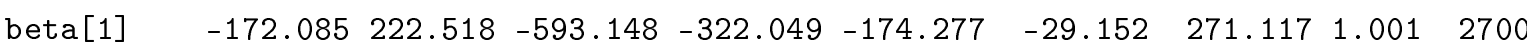

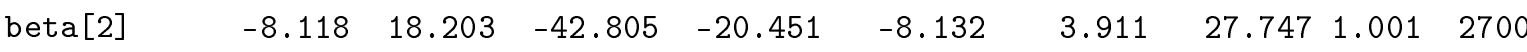

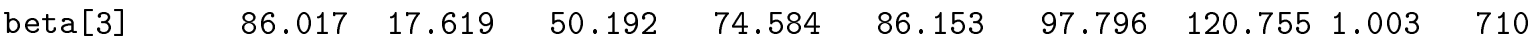

$\begin{array}{llllllllll}\text { beta [4] } & 109.829 & 27.775 & 56.855 & 91.610 & 109.791 & 128.074 & 164.355 & 1.002 & 1300\end{array}$

$\begin{array}{llllllllll}\text { beta[5] } & 49.427 & 17.561 & 14.601 & 37.531 & 49.478 & 61.352 & 83.944 & 1.002 & 1000\end{array}$

$\begin{array}{llllllllll}\text { beta[6] } & 69.788 & 21.848 & 25.700 & 55.362 & 69.934 & 84.013 & 112.446 & 1.002 & 1400\end{array}$

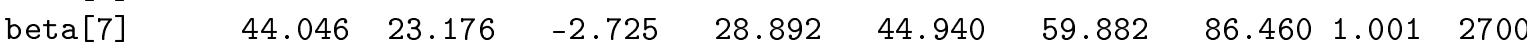




\begin{tabular}{|c|c|c|c|c|c|c|c|c|c|}
\hline [8] & 74.024 & 23.685 & 28.139 & 57.789 & & 89.773 & & 1.002 & 1200 \\
\hline & -25.033 & 3.886 & -71.769 & -41.373 & -25.130 & -9.010 & 22.825 & 1.005 & \\
\hline & 40.482 & 4.296 & -8.234 & 24.490 & 40.748 & 57.269 & 86.565 & 1.002 & 1000 \\
\hline & -40.524 & 4.065 & -86.929 & -56.433 & -40.496 & -24.553 & 6.263 & 1.003 & \\
\hline & 44.448 & 6.808 & -9.310 & 26.548 & 44.400 & 61.754 & 97.419 & 1.001 & 00 \\
\hline & -49.394 & 7.865 & -102.928 & -68.058 & -49.771 & -30.796 & 4.820 & 1.001 & 10 \\
\hline & 100. & 5.930 & 49.666 & 83.252 & 100 & 118. & 149. & 001 & \\
\hline & 10.415 & 5.375 & -38.401 & -6.561 & 10.258 & 27.308 & 60 & 1.001 & 250 \\
\hline & 6.4 & 4.2 & -2 & 3.5 & 395 & & 94 & 001 & \\
\hline & 21.724 & 11.184 & -0.123 & 14.446 & 21.537 & 29.2 & 43. & 1.001 & 270 \\
\hline & 7.430 & 7.490 & -7.468 & 2.607 & 7.491 & 12 . & 21. & 1.001 & 70 \\
\hline & 13.2 & 6.407 & 1.5 & 8 & $12 . \subseteq$ & 17. & 26 . & 001 & 270 \\
\hline ruise & 47.575 & 10.907 & 27.338 & 40.163 & 47.392 & 54.759 & 69.8 & 1.002 & 30 \\
\hline & 36.006 & 6.443 & 23 . & 31.6 & $35 . \varepsilon$ & 40. & 49 . & 000 & 27 \\
\hline $\mathrm{ah}$ & 52.962 & 8.721 & 35.757 & 47.347 & 53.111 & 58.630 & 70.199 & 1.002 & 2 \\
\hline & 7.327 & 4.230 & & 4.032 & & 10. & & 1.002 & 27 \\
\hline & 51.971 & 0.615 & 51.000 & 51.539 & 51.893 & 52.340 & 53.370 & 1.001 & 27 \\
\hline & 13.481 & 9.083 & & 6.195 & 12.127 & & 33. & 1.001 & \\
\hline & 52.069 & 2.358 & 47.864 & 50.446 & 51.911 & 53.554 & 57.051 & 1.002 & \\
\hline viar & 2793.126 & 6.450 & 2782.603 & 2788.464 & 2792.472 & 2796.876 & 2807.700 & 1.001 & 27 \\
\hline
\end{tabular}

For each parameter, n.eff is a crude measure of effective sample size, and Rhat is the potential scale reduction factor (at convergence, Rhat=1).

DIC info (using the rule, $\mathrm{pD}=\operatorname{var}($ deviance) $/ 2$ )

$\mathrm{pD}=20.8$ and $\mathrm{DIC}=2813.9$

DIC is an estimate of expected predictive error (lower deviance is better).

\#\# Finite-population standard deviations

X.mesh.var <- kim.jags\$BUGSoutput\$sims.list [['sd.mesh']] -2

$\mathrm{X}$.protein.var <- kim.jags\$BUGSoutput\$sims.1ist [['sd,protein']]-2

X.chl.var <- kim.jags\$BUGSoutput\$sims.list [['sd.chl'] ] -2

X.temp.var <- kim.jags\$BUGSoutput\$sims.list [['sd.temp']]-2

X.cruise.var <- kim.jags\$BUGSoutput\$sims.list[['sd.cruise'] ] 2

X.int.var <- kim.jags\$BUGSoutput\$sims.list[['sd.int'] ] ${ }^{\sim 2}$

R.var <- kim.jags\$BUGSoutput\$sims.list [['sd.res'] ] -2

$>$ R2.X<- (X.mesh.var + X.cruise.var + X.int.var + X.protein.var + X.chl.var + X.temp.var)/(X.mesh.var + X.cruise.var + X.int.var + X.protein.var + X.ch R2.mesh<-((X.mesh.var)/(X.mesh.var + X.cruise.var + X.int.var + X.protein.var + R.var))

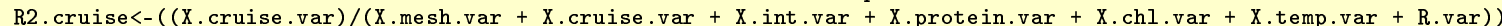

R2.int<- ( X.int.var)/(X.mesh.var + X.cruise.var + X.int.var + X.protein.var + X.chl.var + X.temp.var + R.var))

R2.protein<-((X.protein.var)/(X.mesh.var + X.cruise.var + X.int.var + X.protein.var + X.chl.var + X.temp.var + R.var $))$

R2.chl<-((X.chl.var)/(X.mesh.var + X.cruise.var + X.int.var + X.chl.var + X.chl.var + X.temp.var + R.var $))$

R2.temp<-((X.temp.var $) /(X . m e s h . v a r+X . c r u i s e . v a r+X . i n t . v a r+X . t e m p . v a r+X . c h l . v a r+X . t e m p . v a r+R . v a r))$

R2.resid <- ((R.var)/(X.mesh.var + X.cruise.var + X.int.var + X.protein.var + X.chl.var + X.temp.var + R.var))

a<-cbind(Residual=R.var, 'Temperature'=X.temp.var, 'Chlorophyll'=X.chl.var, 'Protein'=X.protein.var, 'Mesh x Cruise'=X.int.var, Cruise=X.cruise.var, sdtab <- cbind(Name=c('Residuals', 'Temperature', 'Chlorophyll', 'Protein', 'Mesh x Cruise', 'Cruise', 'Mesh'), adply(a, 2, function(x) \{ data.frame $(\operatorname{Mean}=\operatorname{mean}(\mathrm{x})$, Median=median $(\mathrm{x})$, HPDinterval (as $\operatorname{mcmc}(\mathrm{x}))$, HPDinterval $(\operatorname{as} . \mathrm{mcmc}(\mathrm{x}), \mathrm{p}=0.68)$ ) \}))

a $<$-cbind(Residual=R2.resid, 'Temperature'=R2.temp, 'Chlorophyll'=R2.chl, 'Protein'=R2.protein, 'Mesh $x$ Cruise'=R2.int, Cruise=R2.cruise, Mesh=R2.mes R2tab <- cbind(Name=c('Residuals', 'Temperature', 'Chlorophyll', 'Protein', 'Mesh x Cruise', 'Cruise', 'Mesh'), adply(a, 2, function(x) \{ data.frame $(\operatorname{Mean}=\operatorname{mean}(\mathrm{x})$, Median=median $(\mathrm{x})$, HPDinterval (as.mcmc $(\mathrm{x}))$, $\operatorname{HPDinterval}(\operatorname{as} . \operatorname{mcmc}(\mathrm{x}), \mathrm{p}=0.68)$ ) \}))

sdtab\$R2 <- round (R2tab\$Median, 3)

sdtab\$Name <- factor(sdtab\$Name, levels=c('Residuals', 'Temperature', 'Chlorophyll', 'Protein', 'Mesh x Cruise', 'Cruise', 'Mesh'))

sdtab $<-$ subset $($ sdtab, select $=-\mathrm{X} 1)$

print(xtable(sdtab[7:1,], caption='Mean, median, (lower and upper) and highest probability density intervals of finite-population standard deviation

\% latex table generated in R 3.1.0 by xtable 1.7-4 package \% Thu May 7 08:35:45 2015 
Table S4. Mean, median, (lower and upper) and highest probability density intervals of finite-population standard deviations as well as marginal $R^{2}$ approximations.

\begin{tabular}{lrrrrrrr}
\hline Name & Mean & Median & lower & upper & lower.1 & upper.1 & R2 \\
\hline Mesh & 2881.02 & 2820.78 & 1176.67 & 4768.52 & 1758.23 & 3524.99 & 0.30 \\
Cruise & 2382.32 & 2245.97 & 576.33 & 4552.20 & 1109.00 & 3081.09 & 0.23 \\
Mesh x Cruise & 1337.90 & 1282.73 & 472.21 & 2233.68 & 813.63 & 1701.69 & 0.13 \\
Protein & 71.57 & 49.35 & 0.00 & 213.00 & 0.00 & 85.75 & 0.01 \\
Chlorophyll & 216.27 & 168.03 & 0.00 & 585.12 & 0.00 & 264.35 & 0.02 \\
Temperature & 264.21 & 147.07 & 0.00 & 891.61 & 0.00 & 294.42 & 0.02 \\
Residuals & 2701.34 & 2692.84 & 2584.66 & 2823.89 & 2628.70 & 2746.52 & 0.28 \\
\hline
\end{tabular}

p1 <- ggplot (sdtab, aes $(y=$ Name, $x=$ Median $))+$

geom_vline ( $x$ intercept $=0$, linetype='dashed' $)+$

geom_errorbarh (aes (xmin=lower, xmax=upper), height=0)+

geom_errorbarh (aes ( $x$ min=lower.1, xmax=upper .1$)$, height=0, size $=2)+$

geom_point (size $=3$, shape $=21$, fill='white') +

geom_text (aes (label=R2, y=as.numeric (Name)+0.3), position=position_dodge (height=1), size=3)+

scale_x_continuous ('Finite-population standard deviation') +

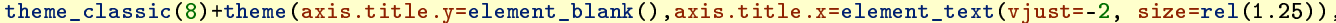
plot.margin=unit $(c(0,0,2,2)$, 'lines'))

$\operatorname{print}(\mathrm{p} 1)$

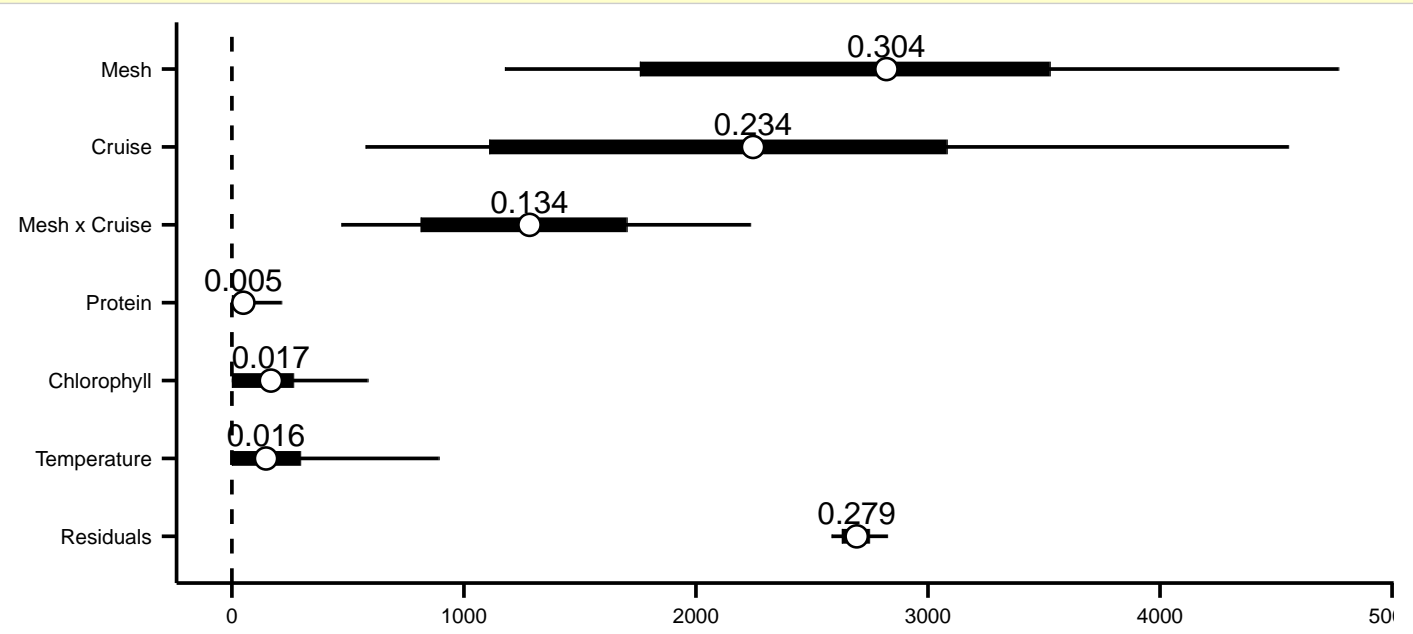

Finite-population standard deviation

Figure T. Median (lower and upper) highest probability density intervals of finite-population standard deviations. $R^{2}$ approximations above medians. 
newdata <- expand.grid(Mesh=levels(kim\$esh), Cruise=levels (kim\$Cruise))

$>$ newdata $\$$ Protein <- mean (kim\$Protein, na.rm=TRUE)

$>$ newdata $\$ \mathrm{Chl}<-$ mean(kim\$Chl, na.rm=TRUE)

$>$ newdata $\$$ Temp <- mean (kim\$Temp, na.rm=TRUE)

$>$ Xmat <- model.matrix ( Mesh*Cruise, data=newdata)

$>$ XXmat <- model.matrix ( $\log ($ Protein $)+\log (\mathrm{Ch} l)+$ Temp, data=newdata) $[,-1]$

$>$ coef <- kim.jags\$BUGSoutput\$sims.list [['beta']]

fit <- coef $\% \%$ t(Xmat) + kim.jags $\$ B U G S o u t p u t \$ s i m s . l i s t[$ ['gamma']] \%*\% t (XXmat)

$>$ library (plyr)

$>$ newdata $<-$ cbind(newdata, adply(fit, 2, function(x) \{

data.frame $(\operatorname{Mean}=\operatorname{mean}(x), \operatorname{Median}=\operatorname{median}(x), \operatorname{HPDinterval}(\operatorname{as} . \operatorname{mcmc}(x)), \operatorname{HPDinterval}(\operatorname{as} . m c m c(x), p=0.68))$ \}))

$>$ \#newdata

\#Comparisons

MCMCsum <- function(x) \{

data.frame (Median=median ( $x$, na.rm=TRUE),

t (quantile ( $x, n a \cdot r m=T R U E)$ )

HPDinterval (as . mcmc ( $x$ )) ,

\} HPDinterval (as . mcmc $(x), p=0.68)$ )

library (multcomp)

ndata $<-$ newdata

ndata $<-$ subset (ndata, Cruise $==$ '1')

Xmat <- $\operatorname{contrMat}(\mathrm{n}=\operatorname{rep}(1$, nrow (ndata)), type="Tukey") \%*\% model.matrix ( Mesh*Cruise, ndata, xlev=list (Mesh=levels (kim $\$$ Mesh), Cruise=levels (kim

$>$ wch <- ldply(strsplit (rownames (Xmat)," - "), as.numeric)

$>\operatorname{rownames}($ Xmat) <- paste (interaction(newdata\$Mesh, newdata $\$ C r u i s e)[w c h[, 1]], "-"$, interaction (newdata $\$$ Mesh, newdata $\$ C r u i s e)[w c h[, 2]])$

pairwise.comps <- coef $\% * \%$ t (Xmat)

$>$ comp.mesh_Cruise1<-adply (pairwise.comps, 2 , MCMCsum)

$>$ ndata $<-$ newdata

ndata $<-$ subset (ndata, Cruise $=={ }^{\prime} 2^{\prime}$ )

Xmat <- contrMat (n=rep (1, nrow (ndata)), type="Tukey") \%*\% model.matrix( Mesh*Cruise, ndata, xlev=list(Mesh=levels(kim\$Mesh), Cruise=levels(kim $\$$ Cruise)

$>$ wch <- ldply(strsplit (rownames (Xmat)," - "), as .numeric)

$>$ rownames (Xmat) <- paste (interaction(newdata $\$$ Mesh, newdata $\$$ Cruise) [wch [,1]], "-", interaction (newdata $\$$ Mesh, newdata $\$ C r u i s e)[w c h[, 2]]$ )

$>$ pairwise.comps <- coef $\% * \%$ t (Xmat)

comp.mesh_Cruise2<-adply (pairwise.comps,2, MCMCsum)

ndata $<-$ newdata

ndata $<-$ subset (ndata, Cruise==' $\left.3^{\prime}\right)$

Xmat <- contrMat (n=rep (1,nrow (ndata)), type="Tukey") \%*\% model.matrix( ${ }^{M e s h * C r u i s e, ~ n d a t a, ~ x l e v=l i s t(M e s h=l e v e l s(k i m ~} \$$ Mesh), Cruise=levels (kim $\$$ Cruise)

$>$ wch <- ldply(strsplit (rownames(Xmat)," - "), as.numeric)

$>\operatorname{rownames}($ Xmat) <- paste (interaction(newdata\$Mesh, newdata $\$ C r u i s e)[w c h[, 1]], "-"$, interaction (newdata $\$$ Mesh, newdata $\$ C r u i s e)[w c h[, 2]])$

pairwise.comps <- coef $\% * \% t$ (Xmat)

comp.mesh_Cruise3<-adply (pairwise.comps, 2, MCMCsum)

ndata $<-$ newdata

ndata $<-$ subset (ndata, Cruise $=={ }^{\prime} 4^{\prime}$ )

Xmat <- contrMat (n=rep(1,nrow(ndata)), type="Tukey") \%*\% model.matrix( Mesh*Cruise, ndata, xlev=list (Mesh=levels(kim $\$$ Mesh), Cruise=levels (kim $\$$ Cruise)

$>$ wch <- ldply(strsplit (rownames(Xmat)," - "), as.numeric)

rownames (Xmat) <- paste(interaction(newdata $\$$ Mesh, newdata $\$ C r u i s e)[w c h[, 1]], "-"$, interaction (newdata $\$$ Mesh, newdata $\$ C r u i s e)[w c h[, 2]]$ )

pairwise.comps <- coef $\% * \%$ t (Xmat)

$>$ comp.mesh_Cruise4<-adply (pairwise.comps, 2 , MCMCsum)

$>$ ndata $<-$ newdata

ndata $<-$ subset (ndata, Cruise $=={ }^{\prime} 5^{\prime}$ )

Xmat <- contrMat (n=rep (1,nrow (ndata)), type="Tukey") \%*\% model.matrix( Mesh*Cruise, ndata, xlev=list (Mesh=levels(kim $\$$ Mesh), Cruise=levels (kim $\$$ Cruise)

$>$ wch <- ldply(strsplit (rownames(Xmat)," - "), as.numeric)

$>$ rownames (Xmat) <- paste (interaction(newdata $\$$ Mesh, newdata $\$ C r u i s e)[w c h[, 1]], "-"$, interaction (newdata $\$ M e s h$, newdata $\$ C r u i s e)[w c h[, 2]]$ )

pairwise.comps <- coef $\% * \%$ t (Xmat)

comp.mesh_Cruise $5<-$ adply (pairwise.comps, 2, MCMCsum)

comp.mesh <- rbind(data.frame (Cruise=1, comp.mesh_Cruise1) data.frame (Cruise $=2$, comp.mesh_Cruise2), data.frame (Cruise $=3$, comp.mesh_Cruise 3 ) data.frame (Cruise $=4$, comp.mesh_Cruise4) data.frame (Cruise $=5$, comp. mesh_Cruise5))

p1 <- ggplot (comp.mesh, aes( $\mathrm{x}=$ Cruise, $\mathrm{y}=$ Median, color $=\mathrm{X} 1))+$ geom_hline (yintercept $=0$, linetype $=$ 'dashed') +

geom_errorbar (aes (ymin=lower, ymax=upper), width=0, position=position_dodge (width=0.5))+ geom_errorbar (aes (ymin=lower.1, ymax=upper.1), width=0, size=2, position=position_dodge $($ width=0.5) ) geom_point (size=3, shape $=21$, fill='white', position=position_dodge $($ width $=0.5))+$

scale_y_continuous('Median effect size') +

\#scale $x$ discrete ('Cruise number') +

scale_color_manual ('Mesh contrasts', breaks=c ('150.1 - 73.1', '350.1 - 73.1', '350.1 - 150.1'), labels=c ('150 vs 73', coord_flip()+theme_classic()+

theme (axis.title. $y=e l e m e n t$ text ( $\operatorname{size}=r e l(1.25)$, vjust $=2)$

axis.title $x=e l$ ement text ( $\operatorname{size}=r e l(1.25)$, vjust=-1), plot.margin=unit $(c(0,0,1,1)$, 'lines'))

ndata $<-$ newdata

ndata<- $\operatorname{subset}($ ndata, Mesh=='73')

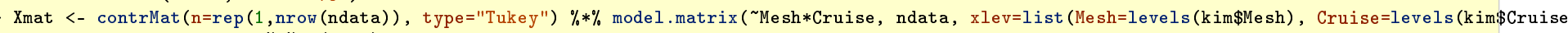
pairwise.comps <- coef $\% * \%$ t (Xmat)

$>$ comp.cruise_Mesh73<-adply (pairwise.comps,2, MCMCsum)

ndata <- newdata

ndata $<-$ subset (ndata, Mesh=='150')

Xmat <- contrMat (n=rep (1,nrow (ndata)), type="Tukey") \%*\% model.matrix( ${ }^{M e s h} *$ Cruise, ndata, xlev=list(Mesh=levels(kim $\$$ Mesh), Cruise=levels (kim $\$$ Cruise)

pairwise.comps <- coef $\% * \%$ t (Xmat) 

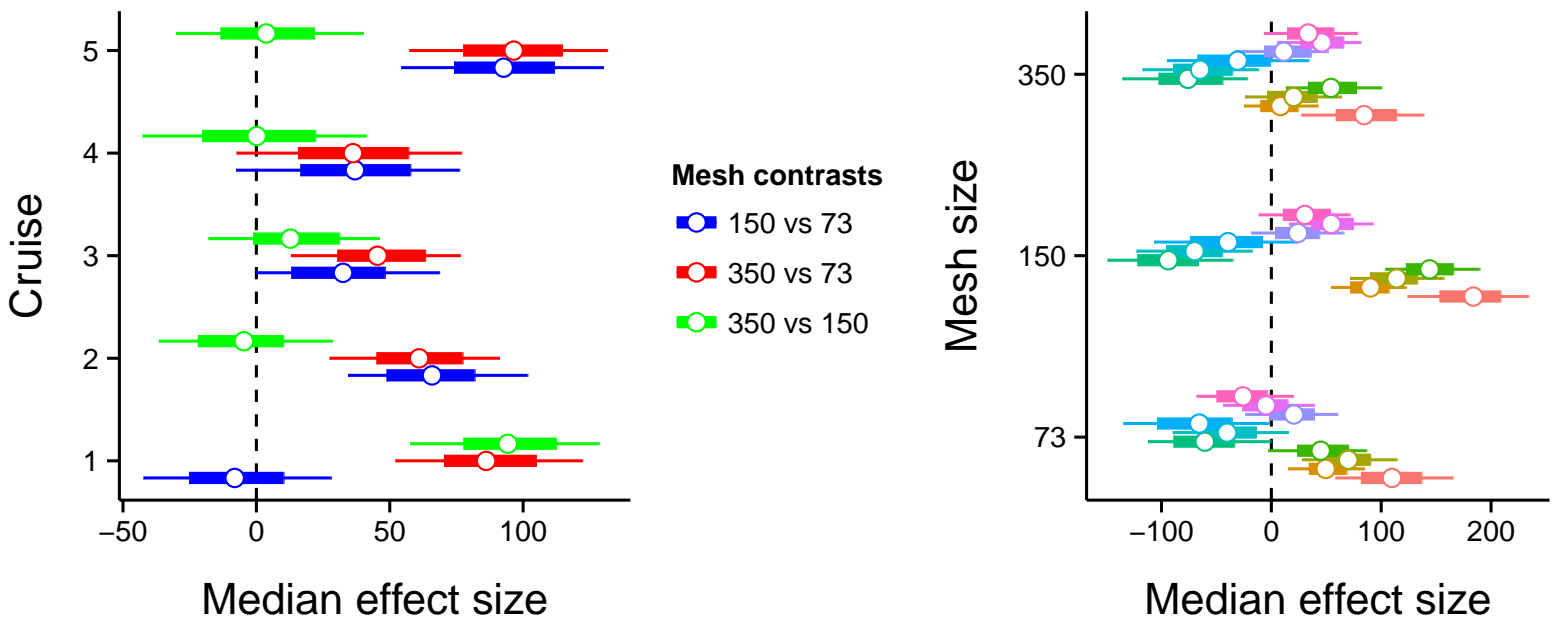

ก- $2-1$

D- $3-1$

- $4-1$

Mesh contrasts

- 150 vs 73

$5-1$

- 350 vs 73

IF 350 vs 150

으 $3-2$

- $-4-2$

1 $5-2$

I $-4-3$

$5-3$

드 $5-4$

Figure $\boldsymbol{U}$. plot of chunk AARSKimcontrasts 


\section{References}

This document was produced from markdown using knitr on R version 3.1.0 (2014-04-10) on a x86_64pc-linux-gnu system.

sessionInfo()

$R$ version 3.1.0 (2014-04-10)

Platform: x86_64-pc-linux-gnu (64-bit)

locale:

[1] LC_CTYPE $=e n \_A U . U T F-8$

[4] LC_COLLATE=en_AU.UTF-8

LC_NUMERIC $=\mathrm{C}$

LC_MONETARY $=$ en_AU.UTF-8

LC_NAME $=\mathrm{C}$

LC_TIME=en_AU.UTF-8

[7] LC_PAPER=en_AU.UTF-8

[10] LC_TELEPHONE $=\mathrm{C}$
LC_MEASUREMENT=en_AU.UTF-8 LC_IDENTIFICATION=C

attached base packages:

[1] splines grid stats graphics grDevices utils datasets methods base

other attached packages:

[1] multcomp_1.3-7 TH.data_1.0-3 survival_2.37-7 mvtnorm_1.0-0 mgcv_1.8-6 nlme_3.1-118

[7] GGally_0.4.8 xtable_1.7-4 plyr_1.8.1 R2jags_0.04-03 rjags_3-13

[13] lattice_0.20-29 gridExtra_0.9.1 ggplot2_1.0.0 knitr_1.9

loaded via a namespace (and not attached):

\begin{tabular}{|c|c|c|c|}
\hline abind_1.4-3 & boot_1.3-13 & colorspace_1.2-4 & digest_0.6.4 \\
\hline evaluate_0.5.5 & formatR_1.0 & gtable_0.1.2 & labeling_0.3 \\
\hline MASS_7.3-35 & Matrix_1.1-4 & munsell_0.4.2 & parallel_3.1.0 \\
\hline proto_0.3-10 & R2WinBUGS_2.1-19 & Rcpp_0.11.3 & reshape_0.8.5 \\
\hline $\begin{array}{l}\text { reshape2_1.4.1.9000 } \\
\text { tools_3.1.0 }\end{array}$ & $\begin{array}{l}\text { sandwich_2.3-2 } \\
\text { zoo_1.7-11 }\end{array}$ & scales_0.2.4 & stringr_0.6.2 \\
\hline
\end{tabular}

Gelman A (2006) Prior distributions for variance parameters in hierarchical models. Bayesian Analysis:515533

Gelman A, Hill J (2007) Data Analysis Using Regression and Multilevel/hierarchical Models. Cambridge University Press, Cambridge, UK

Plummer M (2003) JAGS A program for analysis of Bayesian graphical models using Gibbs sampling. In: Proceedings of the 3rd International Workshop on Distributed Statistical Computing.

R Core Team (2014) R A Language and Environment for Statistical Computing.

Su Y-S, Yajima M (2014) R2jags A Package for Running jags from R.

Team RC (2014) R A Language and Environment for Statistical Computing. 